\title{
The Unruh quantum Otto engine
}

\author{
Enrique Arias, $^{a}$ Thiago R. de Oliveira $^{b}$ and M.S. Sarandy ${ }^{b}$ \\ ${ }^{a}$ Instituto Politécnico, Universidade do Estado do Rio de Janeiro, \\ 28625-570 Nova Friburgo, Brazil \\ ${ }^{b}$ Instituto de Física, Universidade Federal Fluminense, \\ 24210-346 Niterói, Brazil \\ E-mail: earias@iprj.uerj.br, tro@if.uff.br, msarandy@id.uff.br
}

ABSTRACT: We introduce a quantum heat engine performing an Otto cycle by using the thermal properties of the quantum vacuum. Since Hawking and Unruh, it has been established that the vacuum space, either near a black hole or for an accelerated observer, behaves as a bath of thermal radiation. In this work, we present a fully quantum Otto cycle, which relies on the Unruh effect for a single quantum bit (qubit) in contact with quantum vacuum fluctuations. By using the notions of quantum thermodynamics and perturbation theory we obtain that the quantum vacuum can exchange heat and produce work on the qubit. Moreover, we obtain the efficiency and derive the conditions to have both a thermodynamic and a kinematic cycle in terms of the initial populations of the excited state, which define a range of allowed accelerations for the Unruh engine.

KeYwords: Black Holes, Thermal Field Theory

ARXIV EPRINT: 1710.03092 


\section{Contents}

1 Introduction 1

2 Quantum Otto engine 3

3 Quantum Otto engine via Unruh effect 5

3.1 Kinematic cycle of the qubit 5

$\begin{array}{ll}3.2 & \text { Adiabatic expansion of the energy gap }\end{array}$

3.3 Contact with the hot quantum vacuum 8

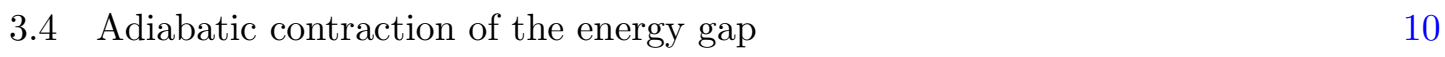

$\begin{array}{ll}\text { 3.5 Contact with the cold quantum vacuum } & 11\end{array}$

4 Quantum vacuum fluctuations effects $\quad 11$

5 Efficiency and cyclicity of the Unruh heat engine 15

6 Conclusions 20

$\begin{array}{ll}\text { A Dynamics of the qubit-vacuum interaction } & 21\end{array}$

B Regularization by Lorentzian profile $\quad 25$

\section{Introduction}

There has been renewed interest in the investigation of the thermodynamics of quantum systems, namely, quantum thermodynamics [1]. A great deal of effort has been devoted to the foundations of the theory, with general discussions about the derivation of the second law in the quantum realm or even the whole theory of thermodynamics from quantum information theory [2]. There is also intense focus on quantum heat engines, Maxwell demons, and advantages of work extraction by global operations over quantum systems [3]. Even though the first works in this direction appeared already in the 1950's [4], it regained interest through the works by Kieu $[5,6]$, where a quantum heat engine is proposed by using as working substance a single two-level system, i.e, a quantum bit (qubit). There, it has been analyzed the quantum Otto cycle, which is the quantum generalization of the classical prototype model of combustion engines. The Otto cycle is composed of two adiabatic and two isochoric steps operating between a hot and a cold reservoir. In order for the machine to produce a finite amount of work, one needs to fulfill the condition $T_{H}>\left(\Delta_{2} / \Delta_{1}\right) T_{C}$, with $T_{H}\left(T_{C}\right)$ the temperature of the hot (cold) reservoir and $\Delta_{1}$ and $\Delta_{2}$ the energy gaps in the qubit Hamiltonian spectrum before and after the adiabatic expansion, respectively. Therefore, for quantum systems, the classical inequality $T_{H}>T_{C}$ has been shown to be 
insufficient for a heat engine to produce work in individual thermodynamic cycles. Another possibility considered in refs. $[5,6]$ is to use a gas of bosons as the thermal reservoir and let the interaction with the system to last a finite time, such that the system does not fully equilibrate with the reservoir. In that case, a cycle can be built by suitably adjusting the initial population of the excited state. These works have been generalized to include different models for the reservoir and also for more complex working substances, e.g. coupled qubits, where quantum correlations such as entanglement may improve the thermal efficiency [7-9].

On the other hand, the relation between quantum thermodynamics, relativistic quantum mechanics, and black hole physics remains little explored. The standard thermodynamics of black holes has been elucidated a long time ago [10], with striking results such as the Bekenstein-Hawking entropy area law [11] and Hawking radiation [12-14]. In this scenario, relativistic effects in quantum thermodynamics may lead to fruitful directions. A particularly relevant phenomenon is the Unruh effect and its implications to the domain of quantum thermodynamics. The Unruh effect predicts the existence of thermal properties to the vacuum state of a quantum field. More specifically, it establishes that the quantum vacuum fluctuations with respect to an accelerated observer in flat space-time exhibit thermal properties with temperature proportional to the observer acceleration $[15,16]$. Even though an experimental realization of the Unruh effect remains as a challenge, it has recently appeared a number of proposals of physical observations, such as in superconducting qubits [17], ion traps [18], quantum metrology [19], oscillating neutrinos [20], and classical electrodynamics [21] (see also ref. [22] for other previous proposals). Here, our aim is to exploit the thermal properties of the vacuum fluctuations predicted by the Unruh effect to model a thermal reservoir and define a relativistic quantum heat engine. We consider as working substance a single qubit linearly coupled to a quantum field prepared in its vacuum state. This is the model used by Unruh and DeWitt in order to detect the quantum vacuum fluctuations effects [16, 23]. The Unruh quantum thermodynamical cycle proposed in our work consists of a series of quantum adiabatic and isochoric processes. During the adiabatic processes, we consider that the qubit undergoes an expansion/contraction of its energy gap due to the influence of some external field. Since the processes are adiabatic, there is no heat exchange between the qubit and the environment. However, the adiabatic expansion/contraction would cost/produce some amount of work. On the other hand, the isochoric processes are defined here by coupling the qubit to the quantum vacuum for finite periods of time and considering two different constant accelerations for the qubit at these stages. In this manner, if the periods of time are very long or infinite lasting we have that, due to the Unruh effect, the qubit would feel the vacuum as a thermal reservoir. For our case, the exact thermal nature of the vacuum fluctuations cannot be recovered, but the finite-time Unruh effect of the vacuum fluctuations can generate a non-trivial response on the accelerated qubit at these stages $[24,25]$. In this way the qubit can absorb some heat from the vacuum fluctuations with no work at these stages. Heat and work are provided by the prescriptions of quantum thermodynamics and are find using a perturbation theory approach in the coupling constant between the qubit and the quantum field. In order to run as a thermal machine, we must also consider the conditions for a closed trajectory of the qubit in real space, since we are dealing with simultaneously thermodynamic and kine- 
matic cycles. The cycle conditions are then derived in terms of the initial populations of the excited state, which define a range of allowed acceleration pairs for the Unruh quantum engine. Throughout the paper, we will adopt natural units, such that the speed of light $c$, the Planck constant $\hbar$, and the Boltzmann constant $k_{B}$ are taken as $c=\hbar=k_{B}=1$.

\section{Quantum Otto engine}

In this section, we briefly review some notions of quantum thermodynamics and quantum heat engines. The basic definitions here are those of work and heat in a quantum process. For a quantum system described by a density operator given by $\rho(t)$ evolving under a time-dependent Hamiltonian $\mathcal{H}(t)$, the energy expectation value $\langle E(t)\rangle=\operatorname{Tr}(\rho(t) \mathcal{H}(t))$ satisfies

$$
\partial_{t}\langle E(t)\rangle=\operatorname{Tr}\left(\partial_{t} \rho(t) \mathcal{H}(t)\right)+\operatorname{Tr}\left(\rho(t) \partial_{t} \mathcal{H}(t)\right) .
$$

We see that the first term on the right hand side of eq. (2.1) is associated with changes in the inner state of the system, which are related to the populations of $\rho(t)$, while the second term has its origin only in changes of the external parameters in $\mathcal{H}(t)$. We thus identify the first as the heat transfer $\langle Q\rangle$ and the second as the work $\langle W\rangle$ realized on the system. This association provides

$$
\langle Q\rangle=\int_{0}^{\mathcal{T}} d t \operatorname{Tr}\left(\frac{d \rho(t)}{d t} \mathcal{H}(t)\right)
$$

and

$$
\langle W\rangle=\int_{0}^{\mathcal{T}} d t \operatorname{Tr}\left(\rho(t) \frac{d \mathcal{H}(t)}{d t}\right),
$$

where $\mathcal{T}$ denotes the total time of evolution and quasi-static processes are adopted. For the processes of interest here, either $\langle Q\rangle$ or $\langle W\rangle$ will be vanishing. Then, from the first law of thermodynamics, this will allow to take these quantities as dependent only on the initial final state of the system. More general cases must be carefully examined (for a review see, e.g., ref. [26]). It is worth observing here that the notion of heat in the quantum thermodynamics domain is entirely compatible with the notions of Boltzmann entropy $S=-\operatorname{Tr}(\rho \ln \rho)$ and that of Boltzmann distribution at equilibrium $\rho=e^{-\mathcal{H} / T} / Z$, where $Z$ is a normalization constant and $T$ is the temperature of the system. By using the definition of heat $\delta Q=T d S$, it is straightforward to show that $\delta Q=\operatorname{Tr}(d \rho \mathcal{H})$, which is equivalent to the definition of mean quantum heat given above.

We now briefly review the Otto quantum engine first proposed in ref. [5]. The classical Otto cycle is composed of two adiabatic processes together with two isochoric processes, which is common in automobile piston engines. In its quantum version, the working substance is a quantum system instead of an ideal gas. Here, we adopt the simplest quantum system, namely, a qubit. The two adiabatic processes, which in the classical engine are provided by the adiabatic expansion and contraction of a piston, here are adiabatic increase and decrease of the upper energy level of the qubit, respectively. The two isochoric process of the classical case are replaced in the quantum version by keeping the system with constant gaps while in contact with a thermal reservoir, which implies in vanishing work. 
Let us now consider a single qubit with eigenlevels $\{|e\rangle,|g\rangle\}$, which are associated with energies $\{\omega, 0\}$, respectively. Hence, by applying the spectral theorem [27], the qubit Hamiltonian can be written as $\mathcal{H}=\omega|e\rangle\langle e|$. In our cycle we consider that, during an adiabatic process, the energy level $\omega$ changes. This change could be caused by some external field or changes in the external conditions as the size of the recipient where the qubit is confined. Here, we only assume it can be done in a smooth way such that the quantum adiabatic theorem is valid [28-30]. The four stages of a quantum Otto cycle are

(i) The qubit begins at the initial state $\rho_{\text {in }}=p|e\rangle\langle e|+(1-p)| g\rangle\langle g|$ with an energy gap $\omega_{1}$ and undergoes an adiabatic expansion to a larger value $\omega_{2}$. This expansion is at the cost of some work over the system. On the other hand, since the process is adiabatic, no heat is exchanged with the environment.

(ii) The qubit in the state $\rho_{\text {in }}$ and with fixed energy gap $\omega_{2}$ is put in contact with a hot bath at temperature $T_{H}$. After some interaction time $\mathcal{T}_{2}$, the qubit ends up at the state $\rho=\left(p+\delta p_{H}\right)|e\rangle\left\langle e\left|+\left(1-p-\delta p_{H}\right)\right| g\right\rangle\langle g|$. Only heat is transfered in this process, with no work performed.

(iii) The qubit at the state $\rho$ is isolated from the hot bath and undergoes an adiabatic compression, which reduces its energy gap from $\omega_{2}$ to $\omega_{1}$. This process is adiabatic, with no change in the populations of the energy levels. Thus, work is performed by the system, with no heat transfered.

(iv) The qubit still with the state $\rho$ is put in contact with a cold reservoir at temperature $T_{C}$. After some interaction time $\mathcal{T}_{1}$, the qubit ends up at the final state $\rho_{\text {fin }}=$ $\left(p+\delta p_{H}+\delta p_{C}\right)|e\rangle\left\langle e\left|+\left(1-p-\delta p_{H}-\delta p_{C}\right)\right| g\right\rangle\langle g|$. Heat is transfered between the qubit and the environment, with no work performed.

One of the novelties in the quantum regime is that the processes are now probabilistic, i.e., while we expect in the classical case that heat flows from the hot bath to the system for every single cycle at stage (ii), namely, $\delta p_{H}>0$, there is in the quantum case a finite probability, for individual cycles, of the opposite to occur, namely, $\delta p_{H}<0$. Thus, it is possible to observe a violation of the behavior expected by the second law of thermodynamics for individual quantum cycles. Naturally, conciliation with the second law is recovered on average over a long sequence of cycles [5]. Claims of violation of the second law have been considered, but they all typically use non-thermal baths (see, e.g., ref. [31]). Note also that the random violation of the second law for individual quantum cycles are due to quantum fluctuations rather than to thermal fluctuations.

The probabilistic character of a quantum engine implies that we need to enforce the cyclicity of the engine through the constraint that $\delta p_{H}+\delta p_{C}=0$, which ensures that the state of the system at the end of stage (iv) is equal to the initial state at the beginning of stage (i). By imposing such a constraint, we obtain that the total amount of work performed by the heat engine is

$$
\Delta W=\delta p_{H}\left(\omega_{2}-\omega_{1}\right)
$$




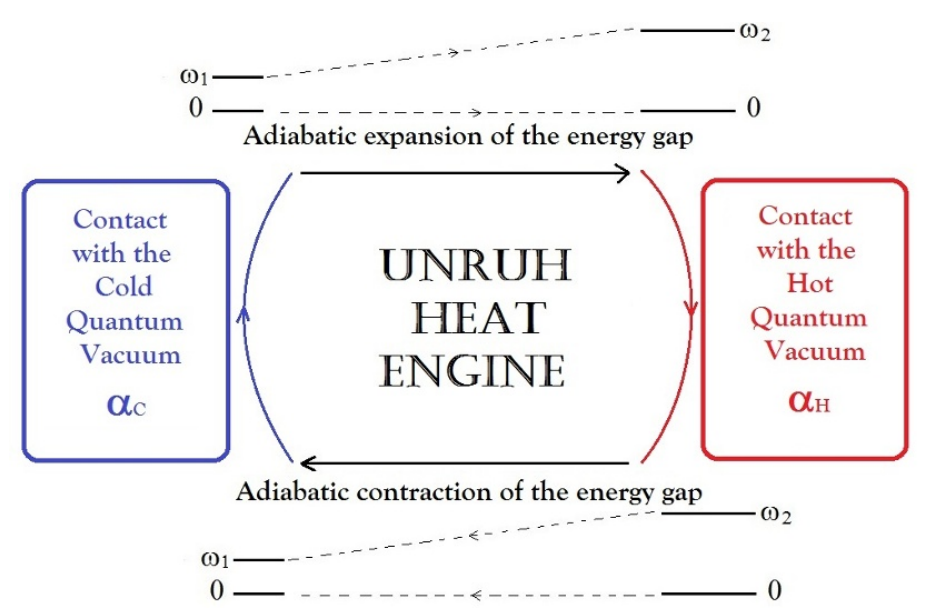

Figure 1. The Unruh quantum thermodynamic cycle.

So it is clear that in order to have a positive work and therefore a heat engine, instead of a refrigerator, we need to require $\delta p_{H}>0$. When the system is let to thermalize with the bath, with $\mathcal{T}_{1}, \mathcal{T}_{2} \rightarrow \infty$, the state of the qubit at the end of stage (ii) is such that $p+\delta p_{H}=$ $\operatorname{Tr}[|e\rangle\langle e| \rho]=1 /\left[1+\exp \left(\beta_{H} \omega_{2}\right)\right]$, where $\beta_{H}=1 / T_{H}$. Similarly at the end of stage (iv), as the system returns to its original state, we have $p=\operatorname{Tr}\left[|e\rangle\langle e| \rho_{\text {fin }}\right]=1 /\left[1+\exp \left(\beta_{C} \omega_{1}\right)\right]$, where $\beta_{C}=1 / T_{C}$. It then follows that the condition for positive work is, on average, $T_{H}>\left(\omega_{2} / \omega_{1}\right) T_{C}$. This is stronger than the classical condition of $T_{H}>T_{C}$. It can be understood by the fact that the system is expected to receive heat from the hot bath if $T_{H}>\omega_{2}$ and to transfer heat to the cold bath if $T_{C}<\omega_{1}$.

\section{Quantum Otto engine via Unruh effect}

We now present the Unruh quantum Otto engine. In this case, the thermal bath comes from the effect of the vacuum fluctuations over the accelerated qubit given by the finite-time Unruh effect. Thus, the thermal reservoir has now a relativistic quantum origin, which is associated with the vacuum fluctuations of a quantum field. This implies that the Unruh effect is able to yield useful work in a cyclic process. As a working substance, we take a single qubit, which is designed to transform heat extracted from the vacuum into work. The stages proposed to the Unruh thermodynamic cycle are illustrated in figure 1.

\subsection{Kinematic cycle of the qubit}

Since the observation of the Unruh effect depends on the state of motion of the qubit as it interacs with the quantum vacuum, a description of the kinematics of the qubit on the whole cycle is required. In this way, in addition to a thermodynamic closed cycle, we also assume a kinematic closed trajectory for the qubit traveling in spacetime. The Unruh effect over closed oscillatory trajectories have been consider in [32]. However, since in our case the conctact of the qubit with the vacuum fluctuations is restricted to some stages of the thermodynamic cycle, we only need to analyze the Unruh effect for a finite part of the qubit trajectory. In this way, the continuous qubit cyclic motion will impose further constraints 


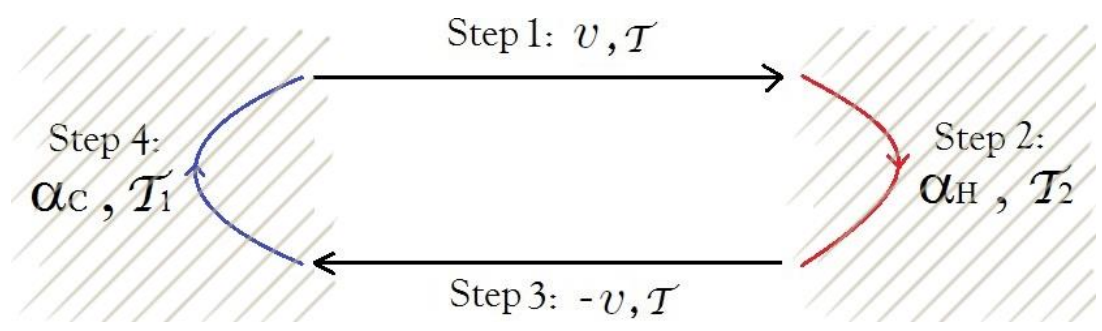

Figure 2. The relativistic kinematic cycle of the qubit. In dashed gray lines are shown the regions of contact between the qubit and the quantum vacuum fluctuations.

in the interaction time with the quantum vacuum, as it will be shown below. Thus the kinematic cycle we analyze is such that, in the first and third step of the cycle, we assume the qubit keeps constant speed, while in the second and fourth steps, it moves with constant acceleration. This is explicitly shown in figure 2. Notice that, while traveling at constant acceleration the qubit is brought into contact with the vacuum fluctuations. On the other hand, while at constant velocity and going through an adiabatic expansion/contraction of the energy gap, there is no contact between the qubit and the quantum field.

In order to assure that there is no change in the state of the system, caused by the vacuum, during the adiabatic processes of the cycle, we should keep the qubit decoupled of the vacuum at these steps. We are thus assuming that one can control the contact between the system and the vacuum in the very same way that one can control the coupling or decoupling between a system and a thermal reservoir in a classical thermal machine. For simplicity we assume a one-dimensional motion of the qubit. Thus, our closed trajectory is composed by the following steps:

- Step 1: constant velocity $v$ during a period of time $\mathcal{T}$.

- Step 2: constant acceleration $\alpha_{H}$ during a period $\mathcal{T}_{2}$. At this step, the qubit accelerates from speed $v$ to $-v$.

- Step 3: constant velocity $-v$ during a period of time $\mathcal{T}$.

- Step 4: constant acceleration $\alpha_{C}$ during a period $\mathcal{T}_{1}$. At this step the qubit accelerates from speed $-v$ to $v$.

It is worth noticing that interaction times $\mathcal{T}_{1}$ and $\mathcal{T}_{2}$ are fixed by the values of $v$ and $\alpha_{H, C}$. More specifically, we will shown that there is a relation between $\alpha_{H}$ and $\alpha_{C}$ in order to ensure that the system returns to its original state after one cycle. For now, we say that it is expected the accelerations (temperatures) satisfy $\alpha_{H}>\alpha_{C}$ to ensure that at step 2 the vacuum behaves like a hot reservoir while at step 4 it acts like a cold reservoir.

The non-trivial motion of the qubit occurs when it is in contact with the vacuum. Indeed, in order for the vacuum to behave as a thermal bath, we have to ensure a motion with constant proper acceleration for the qubit [15]. Therefore, during the stages of contact with the vacuum, we suppose that the qubit moves with constant proper acceleration, as described by the step 2 and step 4 of the kinematics cycle. This would ensure the thermal 


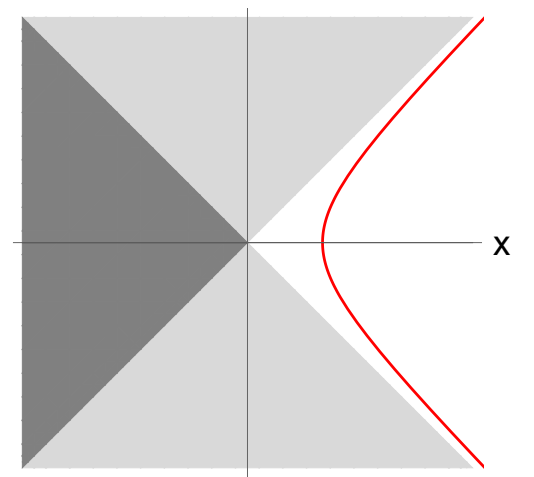

Figure 3. Hyperbolic trajectory of a accelerated qubit. In light gray are shown spacetime regions partially causally disconnected from the qubit. In dark gray is the region totally causally disconnected from the qubit. The white region is the causally accesible region of the qubit, the border of this region represents an event horizon for the accelerated qubit.

behavior of quantum vacuum as predicted by the Unruh effect if a long interaction time is considered. However, even for finite interaction times one can recover a non-null effect over the qubit due to vacuum fluctuations. In general, if one considers a relativistic motion with constant proper acceleration $\alpha$, one finds that the spacetime coordinates of the particle are given by $\chi(\tau)=(t, x)$ where

$$
\begin{aligned}
& t=\frac{1}{\alpha} \sinh (\alpha \tau), \\
& x=\frac{1}{\alpha} \cosh (\alpha \tau),
\end{aligned}
$$

being $\tau$ the particle proper time. This hyperbolic spacetime trajectory of the qubit with constant acceleration is shown in figure 3 .

From eqs. (3.1), the velocity of the qubit is given by

$$
v=\tanh (\alpha \tau) .
$$

Then, we see then that if the qubit velocity begins at $-v$ at time $-\tau$, it will have velocity $v$ at time $\tau$, where

$$
\tau=\frac{1}{\alpha} \operatorname{arctanh}(v)
$$

Then, the time that it takes for the qubit to change from velocity $-v$ to $v$ using a constant acceleration $\alpha$ is $2 \tau=2 \operatorname{arctanh}(v) / \alpha$. Hence, we have that, when the qubit has acceleration $\alpha_{H}$, the interaction time with the vacuum is $\mathcal{T}_{2}=2 \operatorname{arctanh}(v) / \alpha_{H}$, whereas when the qubit has acceleration $\alpha_{C}$ the interaction time with the vacuum is $\mathcal{T}_{1}=2 \operatorname{arctanh}(v) / \alpha_{C}$. Therefore, these are the precise values of the finite interaction times with the vacuum when it would behave as a hot or cold reservoir, respectively.

\subsection{Adiabatic expansion of the energy gap}

Now we describe the first stage of our engine. This process corresponds to an adiabatic expansion of the energy gap, where we assume that the energy gap of the qubit increases 
smoothly in time. Recalling that the qubit Hamiltonian is given by $\mathcal{H}=\omega(t)|e\rangle\langle e|$ and that we consider as the initial state of the qubit the density operator $\rho_{\text {in }}=p|e\rangle\langle e|+(1-p)| g\rangle\langle g|$, it follows that the state of the qubit all along this adiabatic process is kept constant, so $\rho(t)=\rho_{\text {in }}$. Therefore, we obtain that during the adiabatic expansion there is no heat absorbed by the qubit, i.e.

$$
\left\langle Q_{1}\right\rangle=\int_{0}^{\mathcal{T}} d t \operatorname{Tr}\left(\frac{d \rho(t)}{d t} \mathcal{H}(t)\right)=0
$$

However the energy gap expansion of the qubit comes at the cost of work on it, which reads

$$
\begin{aligned}
\left\langle W_{1}\right\rangle & =\int_{0}^{\mathcal{T}} d t \operatorname{Tr}\left(\rho(t) \frac{d \mathcal{H}(t)}{d t}\right), \\
& =\int_{0}^{\mathcal{T}} d t \operatorname{Tr}\left(\rho_{\mathrm{in}} \frac{d(\omega(t)|e\rangle\langle e|)}{d t}\right), \\
& =\int_{0}^{\mathcal{T}} d t \frac{d \omega(t)}{d t} \operatorname{Tr}\left(\rho_{\mathrm{in}}|e\rangle\langle e|\right) .
\end{aligned}
$$

We now use that $\operatorname{Tr}\left(\rho_{\text {in }}|e\rangle\langle e|\right)=p$, where $p$ is the probability of finding the system in the excited state $|e\rangle$. Moreover, considering that the excited state increases its energy level in the adiabatic expansion from $\omega(t=0)=\omega_{1}$ to $\omega(t=\mathcal{T})=\omega_{2}$, we get a positive work given by

$$
\left\langle W_{1}\right\rangle=p\left(\omega_{2}-\omega_{1}\right)
$$

\subsection{Contact with the hot quantum vacuum}

In this part of the cycle we take advantage of the Unruh effect to simulate a thermal bath to the qubit. We take the qubit as interacting with a quantum massless scalar field in its ground state of zero particles, i.e. in its vacuum state $|0\rangle$. Due to the Unruh effect we know that this quantum vacuum fluactuations act in a non-trvial way over the qubit when it moves in an accelerated trajectory, even for finite-time interactions. Therefore the kinematics description of the qubit previously presented is crucial for this part of the cycle. An important remark here is that we suppose that the qubit system is small enough so that it does not affect the state of the scalar field and disturb the quantum vacuum state.

During the interaction of the qubit with the quantum scalar field, we consider that the Hamiltonian of the total qubit-field system is given by

$$
\mathbb{H}=\mathbb{H}_{0}+\mathbb{H}_{\text {int }}
$$

where $\mathbb{H}_{0}$ is the free Hamiltonian and $\mathbb{H}_{\text {int }}$ is the qubit-field interaction Hamiltonian. The Hamiltonian $\mathbb{H}_{0}$ reads

$$
\mathbb{H}_{0}=\mathcal{H}+\mathcal{H}^{\text {field }},
$$

where $\mathcal{H}$ denotes the qubit free Hamiltonian $\mathcal{H}=\omega_{2}|e\rangle\langle e|$, with $\omega_{2}$ the constant gap along this stage, and $\mathcal{H}^{\text {field }}=\int d^{3} x(1 / 2)\left\{\left(\partial_{t} \varphi\right)^{2}+(\nabla \varphi)^{2}\right\}$ is the Klein-Gordon Hamiltonian 
associated to a free massless scalar field $\varphi$. The qubit-field interaction Hamiltonian is given by the linear coupling [23]

$$
\mathbb{H}_{\text {int }}=g m \varphi(\chi(\tau)),
$$

where $g$ is the coupling constant of the interaction, $m$ is the qubit monopole operator, which is given by

$$
m=|e\rangle\langle g|+| g\rangle\langle e|,
$$

and the scalar field $\varphi(\chi(\tau))$ is evaluated on the spacetime point $\chi(\tau)=(t, x)$ where the qubit is located. We suppose at this stage that the qubit has a constant acceleration $\alpha_{H}$, with a spacetime trajectory analog to that given in eq. (3.1).

We consider that the scalar field start its evolution at the vacuum state and the initial state of the qubit-field is the tensor product $\varrho_{\text {in }}=\rho_{\text {in }} \otimes|0\rangle\langle 0|$. We adopt here the interaction picture, where we apply a perturbative approach and a Dyson series to obtain the final state of the qubit up to second order in the small coupling constant $g$. This is shown in details in appendix A. We then show that the final state of the qubit after interacting some time with the vacuum is

$$
\rho(t)=\rho_{\text {in }}+\delta p_{H}(t) \sigma_{3},
$$

where $\sigma_{3}=(|e\rangle\langle e|-| g\rangle\langle g|)$ is the third Pauli matrix and the time $t$ during this vacuum contact is restricted to $-\tau_{2}<t<\tau_{2}$, with $\tau_{2}=\operatorname{arctanh}(v) / \alpha_{H}$. The change in the population of the excited state is given by

$$
\delta p_{H}(t)=g^{2} \int_{-\tau_{2}}^{t} d \tau \int_{-\tau_{2}}^{t} d \tau^{\prime}\left((1-p) e^{-i \omega_{2} \Delta \tau}-p e^{i \omega_{2} \Delta \tau}\right) G_{\alpha_{H}}^{+}\left(\tau, \tau^{\prime}\right),
$$

where $\Delta \tau=\tau-\tau^{\prime}$ and $G_{\alpha_{H}}^{+}\left(\tau, \tau^{\prime}\right)$ is the Green correlation function of the scalar field evaluated at two points of the qubit accelerated trajectory. This vacuum correlation function evaluated on the accelerated trajectory of the qubit would capture the thermal nature of the vacuum fluctuations. Therefore, at some time $t$ during the interaction with the quantum vacuum the density operator of the qubit is given by

$$
\rho(t)=\left(\begin{array}{cc}
p+\delta p_{H}(t) & 0 \\
0 & 1-\left(p+\delta p_{H}(t)\right)
\end{array}\right) .
$$

One can see that the increase of the population of the excited state, eq. (3.12), is caused by the interaction with the vacuum and this would give us, in some limit, a thermal like response, as predicted by the Unruh effect. We also observe that the change in the probability of finding the qubit in its excited state depends on the initial population of this state $p$, on the energy gap $\omega_{2}$ and on the acceleration $\alpha_{H}$, which plays the role of hot temperature in the Unruh quantum machine.

We can then evaluate the average heat and work over the qubit during the contact with the hot quantum vacuum. From the state of the qubit given by eq. (3.13), at any instant in the interval of contact $-\tau_{2}<t<\tau_{2}$, we obtain the mean quantum heat absorbed from the quantum vacuum

$$
\left\langle Q_{2}\right\rangle=\int_{-\tau_{2}}^{\tau_{2}} d t \operatorname{Tr}\left(\frac{d \rho(t)}{d t} \mathcal{H}(t)\right)
$$


Since at this part of the cycle the free qubit Hamiltonian is $\mathcal{H}=\omega_{2}|e\rangle\langle e|$, the mean quantum heat extracted from the vacuum is

$$
\begin{aligned}
\left\langle Q_{2}\right\rangle & =\int_{-\tau_{2}}^{\tau_{2}} d t \operatorname{Tr}\left(\frac{d \rho(t)}{d t} \mathcal{H}\right), \\
& =\int_{-\tau_{2}}^{\tau_{2}} d t \operatorname{Tr}\left(\frac{d\left(\delta p_{H}(t) \sigma_{3}\right)}{d t} \omega_{2}|e\rangle\langle e|\right), \\
& =\omega_{2}\left(\int_{-\tau_{2}}^{\tau_{2}} d t \frac{d\left(\delta p_{H}(t)\right)}{d t}\right) \operatorname{Tr}\left(\sigma_{3}|e\rangle\langle e|\right), \\
& =\omega_{2}\left(\delta p_{H}\left(\tau_{2}\right)-\delta p_{H}\left(-\tau_{2}\right)\right), \\
& =\omega_{2} \delta p_{H},
\end{aligned}
$$

where we have used that, from eq. (3.12), we have $\delta p_{H}\left(-\tau_{2}\right)=0$ and we have also defined the total correction $\delta p_{H}=\delta p_{H}\left(\tau_{2}\right)$, which is given by the finite-time integral

$$
\delta p_{H}=g^{2} \int_{-\tau_{2}}^{\tau_{2}} d \tau \int_{-\tau_{2}}^{\tau_{2}} d \tau^{\prime}\left((1-p) e^{-i \omega_{2} \Delta \tau}-p e^{i \omega_{2} \Delta \tau}\right) G_{\alpha_{H}}^{+}\left(\tau, \tau^{\prime}\right)
$$

Note that $\left\langle Q_{2}\right\rangle$ depends on the energy gap $\omega_{2}$ and on the change $\delta p_{H}$ of the excited level population due to the hot vacuum fluctuations. We will discuss ahead the conditions to ensure that $\delta p_{H}$ and the heat absorbed from the vacuum $\left\langle Q_{2}\right\rangle$ are both positive, so the quantum vacuum here really behaves as a hot reservoir. As at this stage of the cycle, the qubit Hamiltonian is constant $\mathcal{H}=\omega_{2}|e\rangle\langle e|$, which implies that we get no work, i.e.

$$
\left\langle W_{2}\right\rangle=\int_{-\tau_{2}}^{\tau_{2}} d t \operatorname{Tr}\left(\rho(t) \frac{d \mathcal{H}}{d t}\right)=0 .
$$

\subsection{Adiabatic contraction of the energy gap}

We suppose next that the energy gap is contracted by means of external fields. The specific way this contraction occurs is not important, but in order to ensure a closed cycle for the quantum heat engine, we have to get back to the initial energy gap. Then, at this stage we decouple the qubit from the vacuum. Hence, the system is kept in the state

$$
\rho=\left(\begin{array}{cc}
p+\delta p_{H} & 0 \\
0 & 1-\left(p+\delta p_{H}\right)
\end{array}\right) .
$$

Again, the change in the energy levels of the qubit Hamiltonian does not affect the state of the qubit. Then, the state of the qubit remains fixed as in eq. (3.18) all over the adiabatic contraction and there is no heat transfer. On the other hand, the qubit realizes work, which is proportional to the energy gap change and to the population of the excited state. In this way we straightforwardly obtain that, during the adiabatic contraction, heat and work read

$$
\begin{aligned}
\left\langle Q_{3}\right\rangle & =0, \\
\left\langle W_{3}\right\rangle & =\left(p+\delta p_{H}\right)\left(\omega_{1}-\omega_{2}\right),
\end{aligned}
$$

where the energy gap contraction is taken from $\omega_{2}$ to the initial lower value $\omega_{1}$ considered at stage 1 of the quantum cycle. 


\subsection{Contact with the cold quantum vacuum}

Here we consider that the qubit is bring into contact with the quantum vacuum again, but now with acceleration $\alpha_{C}<\alpha_{H}$. So, at this stage, the vacuum plays the role of a the cold bath reservoir. The qubit has a lower energy gap $\omega_{1}$ in this process and its initial state is

$$
\rho=\left(\begin{array}{cc}
p^{\prime} & 0 \\
0 & 1-p^{\prime}
\end{array}\right)
$$

where we have defined $p^{\prime}=p+\delta p_{H}$. Then we have that the final state of the qubit is given by

$$
\rho_{\text {fin }}=\left(\begin{array}{cc}
p^{\prime}+\delta p_{C} & 0 \\
0 & 1-\left(p^{\prime}+\delta p_{C}\right)
\end{array}\right),
$$

where the new change in the probability of the excited state is given by

$$
\delta p_{C}=g^{2} \int_{-\tau_{1}}^{\tau_{1}} d \tau \int_{-\tau_{1}}^{\tau_{1}} d \tau^{\prime}\left(\left(1-p^{\prime}\right) e^{-i \omega_{1} \Delta \tau}-p^{\prime} e^{i \omega_{1} \Delta \tau}\right) G_{\alpha_{C}}^{+}\left(\tau, \tau^{\prime}\right),
$$

as discussed in appendix A. By considering only terms up to second order in the coupling constant and realizing that the first correction $\delta p_{H}$ of eq. (3.16) is already of order two, we can show that

$$
\delta p_{C}=g^{2} \int_{-\tau_{1}}^{\tau_{1}} d \tau \int_{-\tau_{1}}^{\tau_{1}} d \tau^{\prime}\left((1-p) e^{-i \omega_{1} \Delta \tau}-p e^{i \omega_{1} \Delta \tau}\right) G_{\alpha_{C}}^{+}\left(\tau, \tau^{\prime}\right) .
$$

Note that here we have considered that the energy gap of the qubit is $\omega_{1}$ and that the acceleration in this stage of the cycle is lower that previously $\alpha_{C}<\alpha_{H}$. This expression is very similar to that one we have obtained for the case of a hot quantum vacuum. However, in the same way that we must ensure that, during the first contact of the qubit with the quantum vacuum, it absorbed heat, i.e. $\delta p_{H}>0$, here we must require that, in order for the vacuum to behave as a cold reservoir, the qubit have to transfer heat to the vacuum, i.e., $\delta p_{C}<0$. As we will see, these conditions are not independent of each other, since we also need to ensure that during a cycle the qubit comes back to its original state. As before, we can obtain the average quantum heat and the work produced in this part of the cycle, which read

$$
\begin{aligned}
\left\langle Q_{4}\right\rangle & =\omega_{1} \delta p_{C}, \\
\left\langle W_{4}\right\rangle & =0 .
\end{aligned}
$$

After this stage of the cycle, we must impose that the system comes back to its initial state. Then the final state of the qubit, given by eq. (3.21), requires $\delta p_{C}+\delta p_{H}=0$. This cyclicity condition, together with the efficiency of the cycle, are analyzed in the next sections.

\section{Quantum vacuum fluctuations effects}

From our previous results, we have found that the corrections induced by the vacuum fluctuations to the qubit excited state population can be written in a general form as a 
finite-time integral given by

$$
\delta p=g^{2} \int_{-\tau}^{\tau} d t \int_{-\tau}^{\tau} d t^{\prime}\left((1-p) e^{-i \omega \Delta t}-p e^{i \omega \Delta t}\right) G_{\alpha}^{+}\left(t, t^{\prime}\right)
$$

where $\alpha$ is the qubit acceleration, $\omega$ is the qubit energy gap, $p$ is the initial population of the excited state, and $2 \tau$ is the interaction time of the qubit with the vacuum. However, as we need to ensure a kinematically closed cycle for the qubit, we require that the interaction time of the qubit with the vacuum is such that

$$
\tau=\operatorname{arctanh}(v) / \alpha
$$

this precise value ensures us that the qubit enters the vacuum with velocity $v$ and goes out with velocity $-v$, in such a way that its movement in spacetime is continuous and smooth.

The finite-time integral eq. (4.1), is worked out properly in appendix B. In order to avoid spourios effects caused by a sharp switching of the interactions as shown in ref. [33], we consider a continuous smooth regularizator function that limits the interation to a finite domain. A similar procedure have been adopted in [34], in order to consider a finite interaction time between two Unruh-DeWitt detectors with the vacuum. However our method is independent, since we use a Lorentzian regularizator instead of a Gaussian one, we understand that both precedure would be equivalent in some limit. On the other hand, as it is shown in appendix $\mathrm{B}$, the qubit acceleration and the qubit energy gap appear as a single variable, which motivates the definition of the ratio $a \equiv \alpha / \omega$, here called as reduced acceleration. In turn, the correction to the excited state population eq. (B.18), is then written as

$$
\delta p=\delta p(a, p, v)=g^{2}\left((1-2 p) J\left(-\frac{1}{a}, 2 \operatorname{arctanh} v\right)-p \frac{\operatorname{arctanh} v}{2 a}\right) .
$$

We will now numerically analyze the behavior of $\delta p$ given in eq. (4.3) for different regimes of the Unruh machine. The behavior of $\delta p$ can be provided as a function of the reduced acceleration $a=\alpha / \omega$, for different values of initial probabilities $p$ and velocities $v$. The results are illustrated in figure 4 . Note that the corrections $\delta p$ always increase with the acceleration, which plays here the role of the quantum vacuum temperature. In particular, these corrections asymptotically tend to a velocity-dependent constant value for sufficiently high accelerations. Moreover, notice that perturbation theory requires that $|\delta p| \ll p$. This condition may be violated for small $a$, which indicates a breakdown of the perturbative approach. Indeed, as given by eq. (4.2), the interaction time $\tau$ tends to increase as we decrease $a$ for a fixed velocity $v$. Since the integral in eq. (4.1) implies that $|\delta p| \propto g^{2} \omega \tau p$, we have that a large $|\delta p|$ may be yielded in the region of small $a$. This regime is forbidden by perturbation theory and is roughly indicated by the hatched gray areas in figure 4. In fact, by imposing $|\delta p| \ll p$, for $p>0$, we then obtain the condition $g^{2} \omega \tau \ll 1$, which leads to

$$
a \gg g^{2} \operatorname{arctanh}(v) .
$$

Let us discuss in more details the special cases for initial probabilities $p=0,0<p<1 / 2$ 


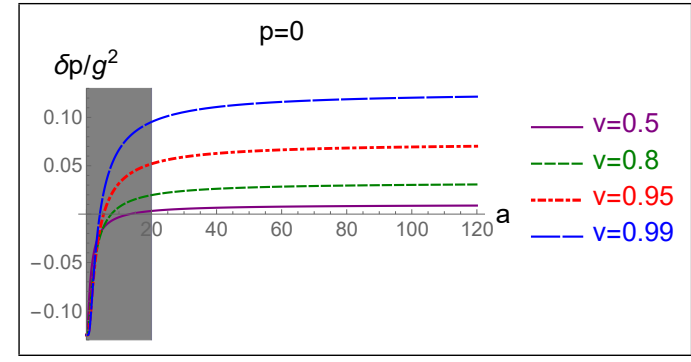

(a) Completely empty excited state.

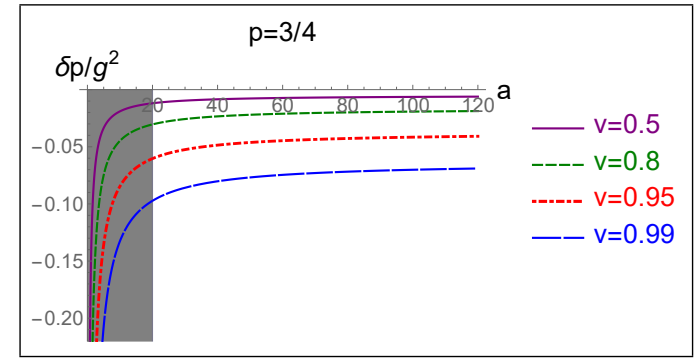

(c) Highly populated excited state.

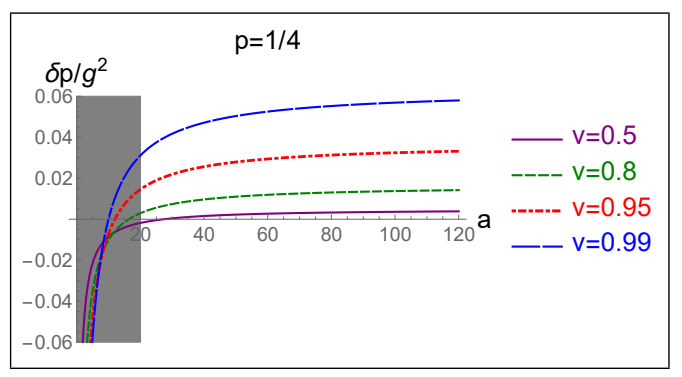

(b) Lowly populated excite state.

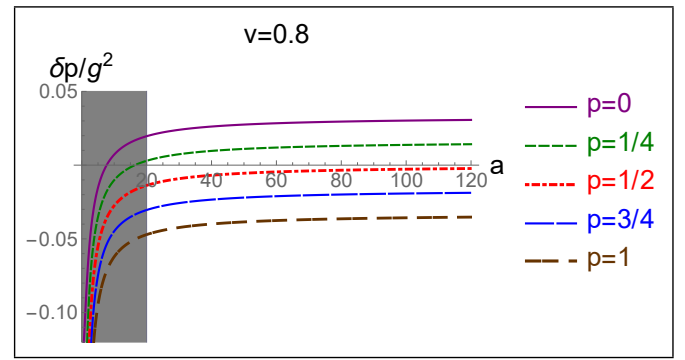

(d) Behavior for fixed velocity.

Figure 4. Behavior of the excited state population correction $\delta p$ as function of the reduced acceleration $a=\alpha / \omega$.

and $1 / 2<p<1$, which are exhibited in figures (4a, 4b, 4c), respectively. For the case $p=0$, we have that the excited state is initially empty and the qubit begins the interaction with the vacuum in its ground state. The breakdown of the perturbative approach appears here in the negative corrections $\delta p$ for small $a$ and this is represented by the gray area. This area, between $a=0$ and $a=20$, encloses one order of magnitude greater that $g^{2} \operatorname{arctanh}(v)$, for $g=1$ and $v=0.99$, as dictated by eq. (4.4). For larger values of $a$, the correction $\delta p$, gets positive and asymptotically increases for larger velocities $v$. This result is consistent with the Unruh effect since, if the system is prepared at the ground state, then the thermal fluctuations created by the Unruh effect would induce an increase in the excited state population. In addition, if $v$ is increased, this means a longer interaction time $\tau$ for a fixed acceleration $a$, which is expected to yield an asymptotically larger correction $\delta p$.

For $p=1 / 4$, as shown in figure (4b), we again see for high accelerations an asymptotically constant value of $\delta p$ that increases as a function $v$, as expected by the Unruh effect. For small acceleration values, we also observe the breakdown of the perturbative approach through the crossings of the curves for distinct values of $v$. The crossings would mean that different interaction times $\tau$ would imply the same excitation $\delta p$, which is a clearly a limitation of the perturbation theory. Moreover, for the point of vanishing acceleration, $a=0$, we see that a negative divergence appears. The origin of this behavior can be traced back to the integral in eq. (4.1), which is proportional to the interaction time interval. Since $\tau \sim 1 / a$, then a divergence comes out for small $a$. This occurs because we would need an infinite time interval to change the velocity from $v$ to $-v$, by moving the qubit with zero acceleration. 


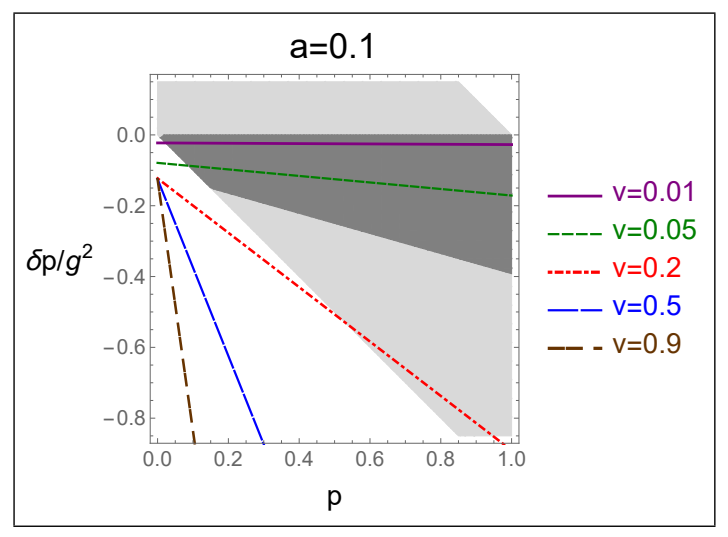

(a) Small accelerations.

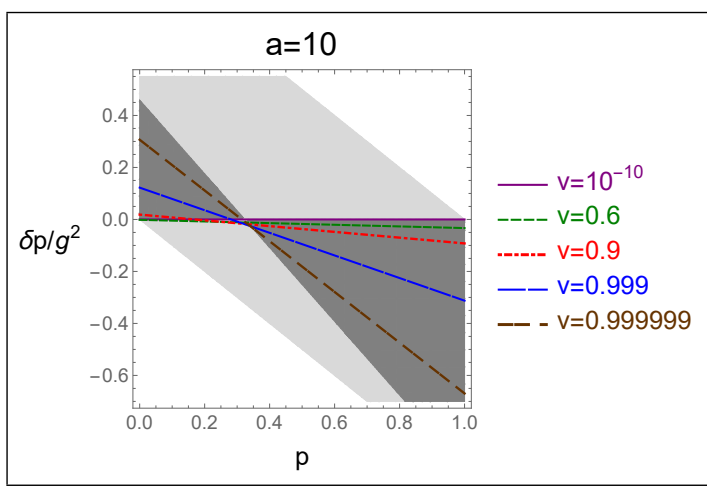

(c) High accelerations.

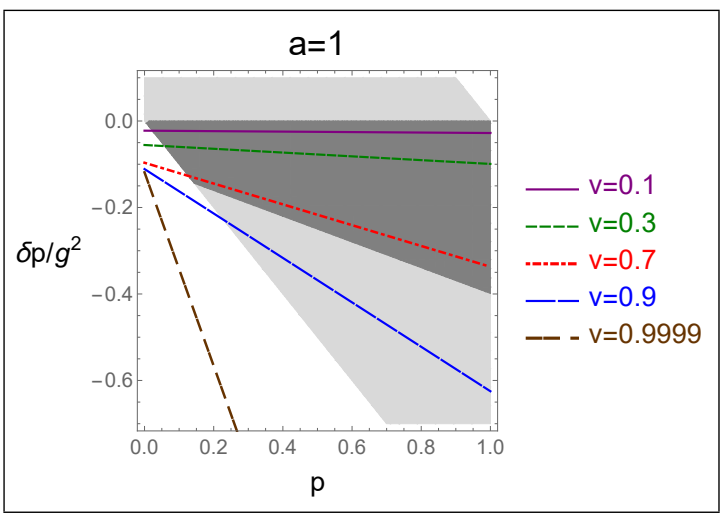

(b) Critical point $\mathrm{a}=1,(\alpha=\omega)$.

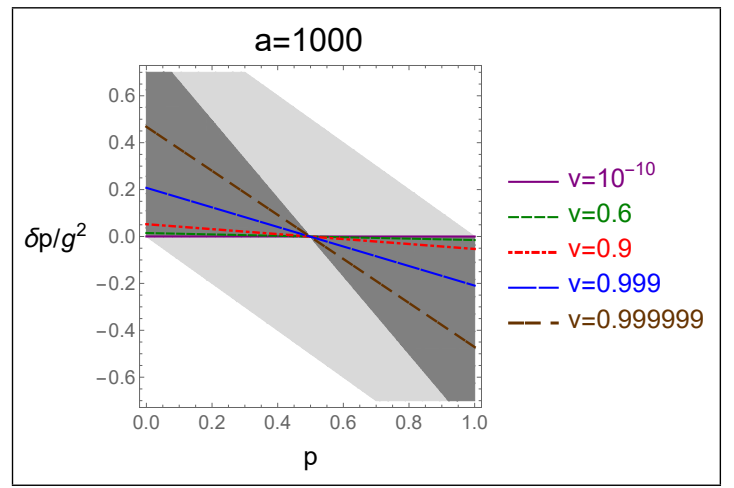

(d) Ultrahigh accelerations.

Figure 5. Behavior of the corrections of the excited state population $\delta p$, in units of $g^{2}$, as function of its initial population, $p$, for different accelerations and velocities. The lighter gray area indicates corrections that obey $0<p+\delta p<1$, where $g=1$ has been considered. The darker gray area indicates points where the perturbative regime is satisfied, ie. $|\delta p| \ll p$.

The case $p=3 / 4$ is presented at figure (4c). We observe that, for this population of the excited state, the vacuum fluctuations would always cause a decrease of the original highly populated excited state. Then, spontaneous and stimulated decay in the qubit energy state would overcome the excitations caused by the Unruh effect. As the acceleration $a$ becomes larger, we have that the corrections $\delta p$ increase, but keeping always negative as a function of $a$.

In figure (4d), we show the behavior of $\delta p$ as a function of $a$ for different initial populations $p$ of the excited state for a fixed velocity. This plot summarizes the previously discussed behaviors for both $p<1 / 2$ and $p>1 / 2$. For the special case $p=1 / 2$, any value of accelerations the Unruh thermal radiation produces a decay in the qubit energy state. However, in the limit of $a \rightarrow \infty$, the corrections are vanishing, i.e. $\delta p=0$. Then, in this case, high accelerations produce a balance on the original equally populated states and no net effect can be detected. In conclusion, it follows that, in order to gain heat from the quantum vacuum, we would require an initial state with $0 \leq p<1 / 2$. Moreover, perturbation theory requires (4.4) as a validation approach. 
We now illustrate in figure 5 the behavior of $\delta p$ as a function of the initial population $p$ of the excited state. As it can be seen, the correction $\delta p$ is linear with $p$, as expected from eq. (4.3). In addition, this linear response is such that $\delta p$ decreases as the initial population $p$ increases. This result is consistent with the Unruh effect over the qubit. For $p \approx 0$, we have an initially lowly populated excited state, so that one expects that the Unruh effect yields an increase of its population, i.e. $\delta p>0$. On the other hand, for $p \approx 1$, the vacuum fluctuations are expected to yield a decay, i.e. $\delta p<0$. Therefore, as $p$ goes from 0 to 1 , one expects the corrections $\delta p$ to decrease and invert its signal, as shown in figure 5 .

In all the plots of figure 5 , we also show a lighter gray area, which represents the allowed values for the corrections $\delta p$ in order to get final positive probabilities less than one. Then, this lighter gray area ensures $0<p+\delta p<1$, for illustration in this area it has been adopted $g=1$. In order to evidence the limits of the perturbative approach we see that there exist values of $\delta p$ that are not in the lighter gray area. However, by using a lower value of the coupling constant as, for example, $g=0.1$, we get that the lighter gray area expands, making better the perturbative results. Then, the lighter gray area is a necessary condition for the validity of perturbation theory. In figure 5 the darker gray area indicates a stronger constraint over perturbation theory, provided by eq. (4.4). This condition gives us the value of the maximum allowed velocity $v_{\max }$ given a value for the acceleration $a$. From eq. (4.4), we adopt $v_{\max }=\tanh \left(a / g^{2}\right)$. In figure (5a), we have analyzed the case for a small reduced acceleration. In this case, we find that only very small values of the velocity remain in the domain of validity. As we increase acceleration, figure (5b), we note that perturbation theory remains valid for a wider range of velocities and only very high velocities $v \sim 1$ are capable to break down the perturbative results. The cases of high and ultrahigh accelerations, shown in figures (5c) and (5d), respectively, are rather robust against violations of the perturbative regime.

\section{Efficiency and cyclicity of the Unruh heat engine}

We can summarize the Unruh heat engine cycle as shown in figure 6. There, we explicitly exhibit the absorbed heat and produced work at each part of the cycle, as well as the changes in the qubit density operator. As we mentioned before, the first contact of the qubit with the quantum vacuum occurs as it moves at a high acceleration $\alpha_{H}$. In this part of the cycle the qubit should absorb heat $\left\langle Q_{2}\right\rangle>0$ from the quantum vacuum and increase the population of the excited state by $\delta p_{H}>0$. After this first contact, the qubit undergoes a contraction of the energy gap. Then, it is put in contact with the quantum vacuum again. During the second contact of the qubit with the quantum vacuum, it should transfer heat $\left\langle Q_{4}\right\rangle<0$ and changes its excited state population by $\delta p_{C}<0$. Therefore, in order for the cycle to yield positive net work, we need to assure both that the vacuum behaves like the hot thermal reservoir in the first contact with the qubit and that it acts as a cold thermal reservoir in the second contact with the qubit. We can explicitly analyze the regimes for these conditions to be obeyed. As we previously mentioned, to obtain a closed cycle, we require that the qubit comes back to its original state as the machine returns to 


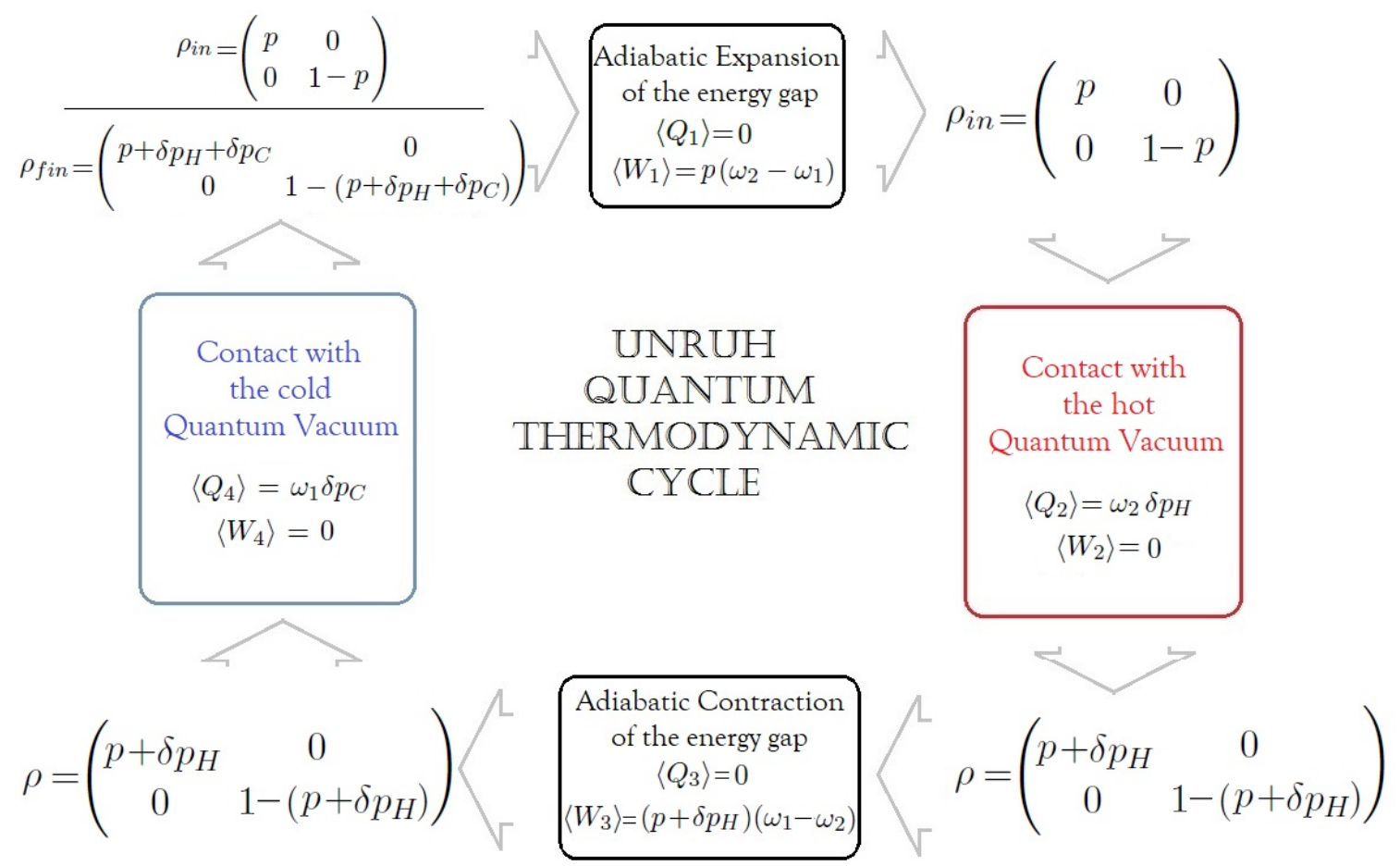

Figure 6. Schematic design of the thermodynamic cycle for the Unruh quantum heat engine. The figure illustrates the different states of the qubit, the absorbed heat, and the produced work at each stage of the cycle.

its first stage, which means

$$
\delta p_{H}+\delta p_{C}=0
$$

Since we expect that $\delta p_{H}>0$, then we should also have $\delta p_{C}<0$. The periodic condition given by eq. (5.1) is equivalent to require that the first law of thermodynamics remains valid on average for our process, as we will see. Consequently, this leads us to the total mean quantum heat absorbed by the qubit from the vacuum

$$
\begin{aligned}
\langle Q\rangle & =\left\langle Q_{2}\right\rangle+\left\langle Q_{4}\right\rangle, \\
& =\omega_{2} \delta p_{H}+\omega_{1} \delta p_{C}, \\
& =\left(\omega_{2}-\omega_{1}\right) \delta p_{H} .
\end{aligned}
$$

On the other hand, work is realized only during the adiabatic expansion or contraction of its energy gap. Hence, the total work produced over the qubit is

$$
\begin{aligned}
\langle W\rangle & =\left\langle W_{1}\right\rangle+\left\langle W_{3}\right\rangle, \\
& =\left(\omega_{2}-\omega_{1}\right) p+\left(\omega_{1}-\omega_{2}\right)\left(p+\delta p_{H}\right), \\
& =\left(\omega_{1}-\omega_{2}\right) \delta p_{H} .
\end{aligned}
$$

Then, as one can see, the net work produced over the qubit and the total heat absorbed from the vacuum satisfies $\langle Q\rangle+\langle W\rangle=0$, as is expected from the first law of thermodynamics. 


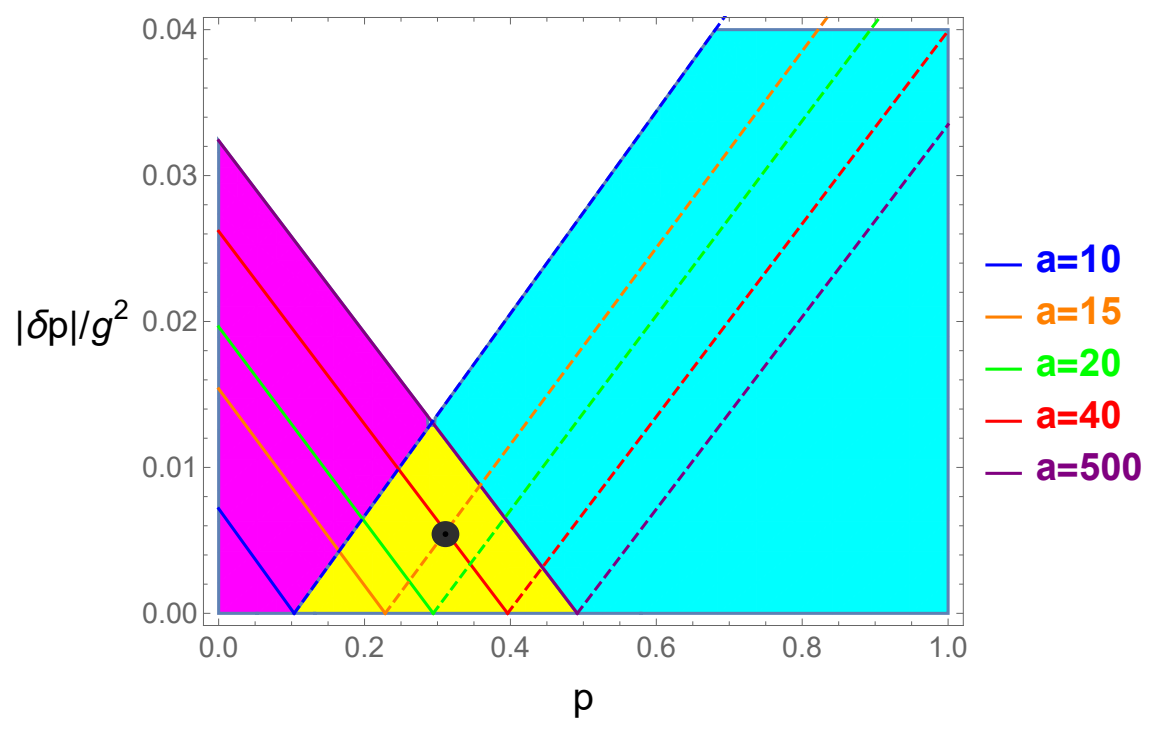

Figure 7. This plot shows the behavior of the absolute value of the correction $|\delta p|$ as function of $p$, for various reduced accelerations $a=\alpha / \omega$ and velocity $v=0.8$. Continuous lines contain points representing positive corrections, with the vacuum behaving as a hot thermal bath. Dashed lines show negative corrections, with the vacuum acting as a cold thermal bath. For the magenta region, the vacuum only acts as a hot reservoir. For the cyan region, the vacuum only acts as a cold reservoir. In the yellow region, we show that the cycle can be closed, since the vacuum acts either as hot or cold reservoir for the same initial value of $p$, with hot or cold depending on the different reduced accelerations associated with the trajectory. The black point intersection defines a triplet $\left(a_{H}, a_{C}, \mathrm{p}\right)$ for which we can define a closed cycle.

Since $\langle W\rangle$ is the work performed over the qubit by external forces, then the work performed by the qubit over the exterior is given by $\langle W\rangle_{\text {ext }}=-\langle W\rangle$. Hence, we define the efficiency $\eta$ as

$$
\eta=\frac{\langle W\rangle_{e x t}}{\left\langle Q_{2}\right\rangle}
$$

which yields

$$
\eta=1-\frac{\omega_{1}}{\omega_{2}}
$$

The efficiency depends only on the ratio of the minimum and maximum energy gaps and is independent of the accelerations (temperatures) we have considered. Moreover, this is the same efficiency found by using a classical thermal bath [5], which suggestes a kind of universal bound independently of the nature of the thermal reservoir.

Let us now, proceed to analyze the kinematic constraints for the cyclic condition of the qubit state, eq. (5.1). By defining the hot $a_{H}$ and cold $a_{C}$ reduced accelerations for the qubit in its two contacts with the vacuum as $a_{H}=\alpha_{H} / \omega_{2}$ and $a_{C}=\alpha_{C} / \omega_{1}$ and using the general result eq. (4.3), then the cyclicity condition given by eq. (5.1) can be written as

$$
\delta p\left(a_{H}, p, v\right)+\delta p\left(a_{C}, p, v\right)=0 .
$$

We also have to ensure that, during the first contact with the vacuum, the qubit has a larger energy gap $\omega_{2}$ and that the qubit absorb heat from the vacuum fluctuations, so that 
$\delta p\left(a_{H}, p, v\right)>0$. This, together with the cyclic condition eq. (5.6), would imply that during the second contact with the vacuum, were the qubit has a lower energy gap $\omega_{1}$, the vacuum acts as a thermal sink, $\delta p\left(a_{C}, p, v\right)<0$. Then, for the same values of $p$ and $v$, but different values of reduced accelerations $a_{H}$ and $a_{C}$, we must ensure that the vacuum acts like a hot or cold thermal bath, respectively. In figure 7 we show the behavior of the absolute value of the correction $|\delta p|$ as a function of the initial probability $p$, for a velocity $v=0.8$ and different reduced accelerations. In solid lines, we show the points where the qubit absorbs heat from the vacuum $(\delta p>0)$ and, in dashed lines, the points where the qubit emits heat to the vacuum $(\delta p<0)$. The yellow region represents the possible values of $a_{H}, a_{C}$ and $p$, where we can find an intersection between solid lines and dashed lines. Therefore, these regions show points corresponding to pairs of accelerations for which, by choosing the initial probability of the excited state as $p$, we will have a closed thermodynamic cycle and extract work from the vacuum. As an illustration, the intersection of the red solid line $\left(a_{H}=40\right)$ and the dashed orange line $\left(a_{C}=15\right)$ occurs at the value $p=0.293$. This triplet satisfies eq. (5.6), with $v=0.8$, allowing for a closed cycle in the Unruh heat engine.

In a more general analysis, eq. (5.6) can be interpreted as an equation for finding the initial population of the excited state $p$, in such a way that the vacuum acts as a hot reservoir for the qubit with reduced acceleration $a_{H}$, while at the same value of $p$, the vacuum acts as a cold reservoir for the qubit with reduced acceleation $a_{C}$. In this manner, we define the critical probability $p_{0}$ that solves eq. (5.6) as the value for our initial population such that the cycle is closed and the qubit returns to its initial state. Then, this probability, which represents intersections of solid and dashed lines in figure 7, depends on the values of the hot and cold reduced accelerations (vacuum temperatures), reading

$$
p_{0}=p_{0}\left(a_{H}, a_{C}, v\right) .
$$

By using eq. (4.3) into eq. (5.6) and defining the function

$$
\mathcal{P}\left(a_{H}, a_{C}, v\right)=\frac{2 a_{H} \cdot a_{C}}{\left(a_{H}+a_{C}\right) \operatorname{arctanh}(v)}\left(J\left(-\frac{1}{a_{H}}, 2 \operatorname{arctanh}(v)\right)+J\left(-\frac{1}{a_{C}}, 2 \operatorname{arctanh}(v)\right)\right),
$$

we can shown that the solution for the initial critical probability $p_{0}$ is

$$
p_{0}=\frac{\mathcal{P}}{1+2 \mathcal{P}} .
$$

The behavior of the solution given by eq. (5.9) for a different pair of reduced accelerations is shown in figure $8 \mathrm{a}$. There, we can see that $0<p_{0}<1 / 2$, as expected from previous discussions. Now, using this solution we can define the correction $\delta \bar{p}_{H}$ such that

$$
\delta \bar{p}_{H}=\delta \bar{p}_{H}\left(a_{H}, a_{C}, v\right)=\delta p\left(a_{H}, p_{0}, v\right) .
$$

The behavior of this function is shown in figure $8 \mathrm{~b}$ ). There, we observe that heat is absorbed from the hot quantum vacuum, $\delta \bar{p}_{H}>0$, and the cycle behaves as a thermal machine instead of a refrigerator, only when $a_{H}>a_{C}$, which implies in the condition

$$
\alpha_{H}>\alpha_{C}\left(\frac{\omega_{2}}{\omega_{1}}\right)
$$




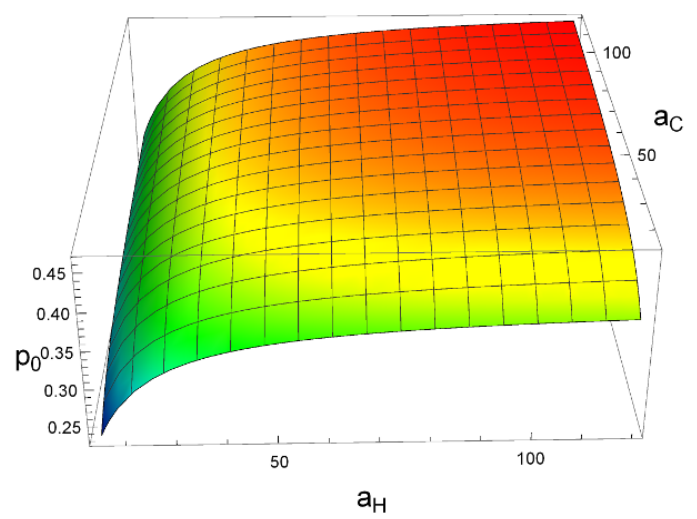

Figure 8 (a). Critical value of the initial probability $p_{0}$ as function of $a_{H}$ and $a_{C}$, for $v=0.8$, such that we have a closed Unruh cycle.

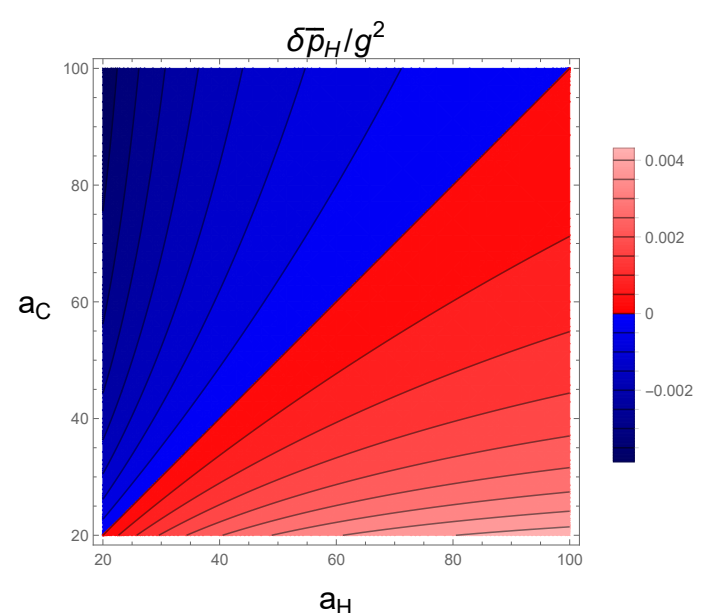

Figure 8 (b). Correction $\delta \bar{p}_{H}$ for different accelerations $a_{H}$ and $a_{C}$. We get positive heat from the quantum vacuum $\delta \bar{p}_{H}>0$, only for $a_{H}>a_{C}$.

This relation is analog to the result find in [5], however in our case the role of temperatures is interpreted by the accelerations of the qubit when interacts with the vacuum fluctuations. This is in accordance with the thermal nature of the quantum vacuum predicted by Unruh. This condition, eq. (5.11), is stronger that the classical relation $\alpha_{H}>\alpha_{C}$, and establish which gradients of accelerations (temperatures) could be used in order to run a quantum heat engine. Note also that we have find the initial critical probability, given by eq. (5.9), in order to run the quantum thermal machine. Finally, by defining the change due to the cold reservoir as $\delta \bar{p}_{C}=\delta p\left(a_{C}, p_{0}, v\right)$, we get that $\delta \bar{p}_{C}=-\delta \bar{p}_{H}$, so in the domain defined by eq. (5.11) we obtain that the vacuum acts like a cold reservoir when the qubit is prepare to have initial critical probability $p_{0}$ and has a small reduced acceleration $a_{C}$. All this ensure us that the cycle behaves as a heat engine instead of a refrigerator.

In order to conclude our analysis, we will realize a comparison between the works performed by Unruh quantum Otto engine and the usual quantum heat engine relying on a classical thermal bath. For a classical thermal bath, we have that the work provided by the machine is given by $W_{c l}=\left(\omega_{2}-\omega_{1}\right) \delta p_{c l}$, where

$$
\delta p_{c l}=\frac{1}{1+e^{\beta_{H} \omega_{2}}}-\frac{1}{1+e^{\beta_{C} \omega_{1}}},
$$

is the change in the population of the excited state when the qubit pass through the hot thermal bath. Here we suppose that the qubit begins this contact with an equilibrium distribution, which has a temperature given by the cold reservoir and, after this contact, the qubit state has an equilibrium distribution with temperature given by the hot reservoir. We compare our result $W=\left(\omega_{2}-\omega_{1}\right) \delta \bar{p}_{H}$ with the classical result when the temperatures $T_{H, C}=1 / \beta_{H, C}$ coincide with the accelerations $\alpha_{H, C}$ we used here. Then, in this case, we can rewrite the classical correction in terms of the reduced accelerations

$$
\delta p_{c l}\left(a_{H}, a_{C}\right)=\frac{1}{1+e^{1 / a_{H}}}-\frac{1}{1+e^{1 / a_{C}}} .
$$




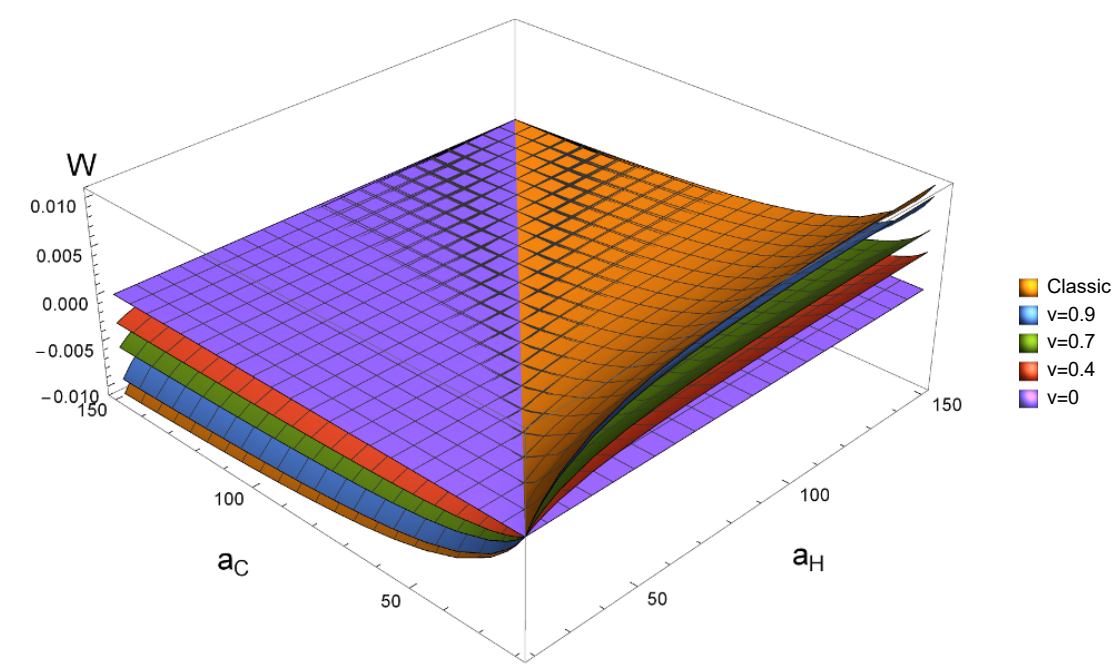

Figure 9. Comparison between the work performed by the Unruh heat engine with different qubit velocities and the usual quantum heat engine with a classical thermal reservoir. Here we have used $\omega_{2}-\omega_{1}=1$.

Since, our correction $\delta \bar{p}_{H}\left(a_{H}, a_{C}, v\right)$ also depends on the velocity of the qubit in the kinematic cycle, we can compare this correction for different values of the qubit velocity versus the classical thermal bath correction. This is shown in figure 9. There we can see that, as we increase the qubit velocity $v$ in the quantum Unruh heat engine, the results approach the expected result of a classical thermal bath. This can be understood in terms of the finite interaction time with the vacuum. Since we are dealing with a finite trajectory in space-time, the interaction time of the qubit with the vacuum fluctuations is finite and given by $\mathcal{T}=2 \operatorname{arctanh}(v) / \alpha$. So, as we increase the qubit velocity, the interaction time with the vacuum fluctuations also increases and we approach the exact thermal nature of the vacuum predicted by Unruh. It is worth noting here that perturbation theory constrains us to small values of $\mathcal{T}$, so very small values of $\alpha$ as well as ultrahigh velocities $v \approx 1$ must be avoided.

\section{Conclusions}

We have introduced a relativistic quantum thermal machine based on the Unruh effect. This is achieved by taking the quantum vaccuum as a thermal reservoir. By using quasistatic processes and perturbation theory, we are then able to establish the conditions over the initial excitation probability $p_{0}$ and qubit accelerations $a_{H}$ and $a_{C}$ for ensuring closed cycles, both from the thermodynamic and kinematic point of view. More specifically, we determine sets of triplets $\left(p_{0}, a_{H}, a_{C}\right)$ such that a closed cycle emerges. Moreover, we have also shown that the classical efficiency of the Otto cycle persists in the relativistic regime, suggesting a universal bound as long as quasi-static processes are adopted. On the other hand, work is nontrivially affected by kinematics, being dependent on the velocities and accelerations of the qubit throughout its time evolution. This analysis has been analytically provided via perturbation theory, with its limitations delineated. 
Even though realizations of the Unruh effect are still challenging, the thermal machine proposed here opens the possibility of posing relativistic quantum themodynamics in an experimental setting for quantum motors and refrigerators as long as alternative approaches [17-22] for probing the Unruh effect are well-succeeded. As further developments, it remains the extension of our approach to the non-equilibrium regime in open systems, e.g., with the entropy production taken into account [35]. Moreover, it is still promising the investigation of the role of correlations, such as entanglement, for the machine efficiency. These topics are left for future research.

\section{Acknowledgments}

E.A. would like to thank Guillermo Dueñas for many useful discussions while this work has been prepared and to FAPERJ for financial support. T.R.O. would like to thank CNPq-Brazil for financial support. M.S.S. acknowledges support from CNPq-Brazil (No.303070/2016-1) and FAPERJ (No 203036/2016). The authors also acknowledge the Brazilian National Institute for Science and Technology of Quantum Information (INCT-IQ).

\section{A Dynamics of the qubit-vacuum interaction}

During the interaction of the qubit with the quantum scalar field we have that the Hamiltonian of the total qubit-field system is given by

$$
\mathbb{H}=\mathbb{H}_{0}+\mathbb{H}_{\text {int }},
$$

the free Hamiltonian $\mathbb{H}_{0}$ is the part that does not consider interaction between the qubit and the field

$$
\mathbb{H}_{0}=\mathcal{H}+\mathcal{H}^{\text {field }},
$$

where $\mathcal{H}^{\text {field }}=\int d^{3} x(1 / 2)\left\{\left(\partial_{t} \varphi\right)^{2}+(\nabla \varphi)^{2}\right\}$ is the Klein-Gordon Hamiltonian associated to a free massless scalar field $\varphi$ and $\mathcal{H}$ is the qubit free Hamiltonian given by $\mathcal{H}=\omega|e\rangle\langle e|$. The qubit-field interaction Hamiltonian is given by

$$
\mathbb{H}_{\text {int }}=g m \varphi(\chi(\tau)),
$$

where $g$ is a small coupling constant of the interaction, $m$ is the qubit monopole operator given by

$$
m=|e\rangle\langle g|+| g\rangle\langle e|,
$$

and $\varphi(\chi(\tau))$ is the scalar field evaluated on the spacetime point $\chi(\tau)=(t, x)$, where the qubit is located. These coordinates are given by eq. (3.1), with the qubits moving with constant acceleration $\alpha$. Let us denote $\varrho$ the total density operator of the qubit-field system. Then this operator satisfies the quantum Liouville's equation

$$
i \frac{d \varrho(t)}{d t}=[\mathbb{H}, \varrho] .
$$


The qubit is prepared in the mixed state $\rho_{\text {in }}$ and the scalar field starts at the pure vacuum state $|0\rangle$. The initial density operator of the total system is taken as the tensor product $\varrho_{\text {in }}=\rho_{\text {in }} \otimes|0\rangle\langle 0|$. In order to simplify the dynamics, we move to the interaction picture. Then, we define the total density operator in the interaction picture $\varrho_{I}(t)$ as a pull-back over $\varrho(t)$, yielding

$$
\varrho_{I}(t)=\mathbb{U}_{0}^{-1}\left(t, t_{0}\right) \varrho(t) \mathbb{U}_{0}\left(t, t_{0}\right),
$$

where $\mathbb{U}_{0}\left(t, t_{0}\right)$ is the total evolution operator when there is no interaction between the qubit and the field. Therefore, this operator satisfies

$$
i \frac{\partial \mathbb{U}_{0}\left(t, t_{0}\right)}{\partial t}=\mathbb{H}_{0} \mathbb{U}_{0}\left(t, t_{0}\right)
$$

The dynamics of the density operator in the interaction picture then reads

$$
i \frac{d \varrho_{I}(t)}{d t}=\left[\mathbb{H}_{\mathrm{int}}^{I}, \varrho_{I}(t)\right],
$$

where $\mathbb{H}_{\text {int }}^{I}$ is the interaction Hamiltonian in the interaction picture. As before, the initial condition is given by $\varrho_{I}\left(t_{0}\right)=\varrho\left(t_{0}\right)=\varrho_{\text {in }}=\rho_{\text {in }} \otimes|0\rangle\langle 0|$. Hence, the solution to eq. (A.8) is given in terms of a Dyson series

$$
\varrho_{I}(t)=\varrho_{\text {in }}-i \int_{t_{0}}^{t} d \tau\left[\mathbb{H}_{\text {int }}^{I}(\tau), \varrho_{\text {in }}\right]-\int_{t_{0}}^{t} d \tau \int_{t_{0}}^{\tau} d \tau^{\prime}\left[\mathbb{H}_{\text {int }}^{I}(\tau),\left[\mathbb{H}_{\text {int }}^{I}\left(\tau^{\prime}\right), \varrho_{\text {in }}\right]\right]+\ldots
$$

We use now the temporal order product operator $T$, which acts as

$$
\begin{aligned}
T\left\{\left[\mathbb{H}_{\text {int }}^{I}(\tau),\left[\mathbb{H}_{\text {int }}^{I}\left(\tau^{\prime}\right), \varrho_{\text {in }}\right]\right]\right\}= & \theta(\Delta \tau)\left[\mathbb{H}_{\text {int }}^{I}(\tau),\left[\mathbb{H}_{\text {int }}^{I}\left(\tau^{\prime}\right), \varrho_{\text {in }}\right]\right] \\
& +\theta(-\Delta \tau)\left[\mathbb{H}_{\text {int }}^{I}\left(\tau^{\prime}\right),\left[\mathbb{H}_{\text {int }}^{I}(\tau), \varrho_{\text {in }}\right]\right],
\end{aligned}
$$

with $\Delta \tau=\tau-\tau^{\prime}$. Then, we can write $\varrho_{I}(t)$ as

$$
\begin{aligned}
\varrho_{I}(t)= & \varrho_{\text {in }}-i \int_{t_{0}}^{t} d \tau\left[\mathbb{H}_{\text {int }}^{I}(\tau), \varrho_{\text {in }}\right] \\
& -\frac{1}{2} \int_{t_{0}}^{t} d \tau \int_{t_{0}}^{t} d \tau^{\prime} T\left\{\left[\mathbb{H}_{\text {int }}^{I}(\tau),\left[\mathbb{H}_{\text {int }}^{I}\left(\tau^{\prime}\right), \varrho_{\text {in }}\right]\right]\right\}+\ldots
\end{aligned}
$$

We consider here that the contact with the vacuum occurs at general times $-\bar{\tau}<\tau<\bar{\tau}$, where we have that $\bar{\tau}=\operatorname{arctanh}(v) / \alpha$. From eq. (A.11), we need to express the interaction Hamiltonian, $\mathbb{H}_{\text {int }}=g m \varphi(\chi(\tau))$, in the interaction picture. Given an operator in the Schrodinger picture $\mathcal{O}_{S}$, its version in the interaction picture is

$$
\mathcal{O}_{I}(t)=\mathbb{U}_{0}^{-1}\left(t, t_{0}\right) \mathcal{O}_{S} \mathbb{U}_{0}\left(t, t_{0}\right) .
$$

Therefore the interaction Hamiltonian in the interaction picture is given by

$$
\mathbb{H}_{\mathrm{int}}^{I}(\tau)=g \mathbb{U}_{0}^{-1}\left(\tau, t_{0}\right) m \varphi(\chi(\tau)) \mathbb{U}_{0}\left(\tau, t_{0}\right),
$$

where we have defined

$$
\mathbb{U}_{0}\left(\tau, t_{0}\right)=e^{-i \mathbb{H}_{0}\left(\tau-t_{0}\right)} .
$$


Since $\mathbb{H}_{0}=\mathcal{H}+\mathcal{H}^{\text {field }}$ is time independent and $\left[\mathcal{H}, \mathcal{H}^{\text {field }}\right]=0$, it follows

$$
\mathbb{U}_{0}\left(\tau, t_{0}\right)=\mathcal{U}\left(\tau, t_{0}\right) \mathcal{U}^{\text {field }}\left(\tau, t_{0}\right),
$$

where $\mathcal{U}\left(\tau, t_{0}\right)=e^{-i \mathcal{H}\left(\tau-t_{0}\right)}$ and $\mathcal{U}^{\text {field }}\left(\tau, t_{0}\right)=e^{-i \mathcal{H}^{\text {field }}\left(\tau-t_{0}\right)}$. Therefore

$$
\mathbb{H}_{\text {int }}^{I}(\tau)=g m_{I}(\tau) \varphi_{I}(\chi(\tau)),
$$

where we have defined

$$
\begin{aligned}
m_{I}(\tau) & =\mathcal{U}^{-1}\left(\tau, t_{0}\right) m \mathcal{U}\left(\tau, t_{0}\right), \\
\varphi_{I}(\chi(\tau)) & =\mathcal{U}^{\text {field }}{ }^{-1}\left(\tau, t_{0}\right) \varphi(\chi(\tau)) \mathcal{U}^{\text {field }}\left(\tau, t_{0}\right) .
\end{aligned}
$$

Since operators in the interaction picture, eq. (A.17), evolve as free operators without taking into account the influence between the field and the quibt, then the scalar field $\varphi_{I}(\chi(\tau))$ represents the free scalar field evaluated at the position of the qubit. The vacuum expected values of products of this operator give us the free Green correlation functions of the field. For instance, the free Wightman function of the free scalar field is given by

$$
G^{+}\left(\chi, \chi^{\prime}\right)=\left\langle 0\left|\varphi_{I}(\chi) \varphi_{I}\left(\chi^{\prime}\right)\right| 0\right\rangle .
$$

Let us now explicitly work the monopole operator at the interaction picture $m_{I}(\tau)$. As the initial time during the contact with the vacuum is $t_{0}=-\bar{\tau}$ and the Hamiltonian of the qubit $\mathcal{H}=\omega|e\rangle\langle e|$ is time independent, then

$$
\begin{aligned}
\mathcal{U}_{0}\left(\tau, t_{0}\right) & =\mathcal{U}_{0}(\tau,-\bar{\tau}), \\
& =e^{-i \omega_{2}(\tau+\bar{\tau}) \tilde{\sigma}}, \\
& =\mathbb{I}+\left(e^{-i \omega_{2}(\tau+\bar{\tau})}-1\right) \tilde{\sigma}, \\
& =\left(\begin{array}{cc}
e^{-i \omega_{2}(\tau+\bar{\tau})} & 0 \\
0 & 1
\end{array}\right) .
\end{aligned}
$$

The monopole operator is given by $m=|e\rangle\langle g|+| g\rangle\langle e|$. Then its matrix representation is

$$
m=\left(\begin{array}{ll}
0 & 1 \\
1 & 0
\end{array}\right) \text {. }
$$

In the interaction picture $m_{I}(\tau)$ reads

$$
\begin{aligned}
m_{I}(\tau) & =\mathcal{U}^{-1}(\tau,-\bar{\tau}) m \mathcal{U}(\tau,-\bar{\tau}), \\
& =\left(\begin{array}{cc}
0 & e^{i \omega_{2}(\tau+\bar{\tau})} \\
e^{-i \omega_{2}(\tau+\bar{\tau})} & 0
\end{array}\right) .
\end{aligned}
$$

The state of the qubit-field system at the instant $-\bar{\tau}<t<\bar{\tau}$ after the interaction have begun is, up to second order in perturbation theory, given by

$$
\begin{aligned}
\varrho_{I}(t)= & \varrho_{\text {in }}-i g \int_{-\bar{\tau}}^{t} d \tau\left[m_{I}(\tau) \varphi_{I}(\chi(\tau)), \varrho_{\text {in }}\right] \\
& -\frac{g^{2}}{2} \int_{-\bar{\tau}}^{t} d \tau \int_{-\bar{\tau}}^{t} d \tau^{\prime} T\left\{\left[m_{I}(\tau) \varphi_{I}(\chi(\tau)),\left[m_{I}\left(\tau^{\prime}\right) \varphi_{I}\left(\chi\left(\tau^{\prime}\right)\right), \varrho_{\text {in }}\right]\right]\right\} .
\end{aligned}
$$


Since we are interested only in the qubit state independently of the field, we perform a partial trace over the field degrees of freedom on the total state of the qubit-field system $\varrho_{I}(t)$, which yields

$$
\rho_{I}(t)=\operatorname{Tr}_{\text {field }} \varrho_{I}(t) .
$$

By taking into account that $\varrho_{\text {in }}=\rho_{\text {in }} \otimes|0\rangle\langle 0|$, the partial traces produce

$$
\begin{aligned}
\operatorname{Tr}_{\text {field }} \varrho_{\text {in }}= & \rho_{\text {in }}, \\
\operatorname{Tr}_{\text {field }}\left[m_{I}(\tau) \varphi_{I}(\chi(\tau)), \varrho_{\text {in }}\right]= & 0, \\
\operatorname{Tr}_{\text {field }}\left[m_{I}(\tau) \varphi_{I}(\chi(\tau)),\left[m_{I}\left(\tau^{\prime}\right) \varphi_{I}\left(\chi\left(\tau^{\prime}\right)\right), \varrho_{\text {in }}\right]\right]= & m_{I}(\tau) m_{I}\left(\tau^{\prime}\right) \rho_{\text {in }} G_{\alpha}^{+}\left(\tau, \tau^{\prime}\right) \\
& +\rho_{\text {in }} m_{I}\left(\tau^{\prime}\right) m_{I}(\tau) G_{\alpha}^{+}\left(\tau, \tau^{\prime}\right) \\
& -m_{I}(\tau) \rho_{\text {in }} m_{I}\left(\tau^{\prime}\right) G_{\alpha}^{+}\left(\tau, \tau^{\prime}\right) \\
& -m_{I}\left(\tau^{\prime}\right) \rho_{\text {in }} m_{I}(\tau) G_{\alpha}^{+}\left(\tau, \tau^{\prime}\right),
\end{aligned}
$$

where we have evaluated the Wightman functions of the scalar field at different points of the qubit trajectory, denoting them as $G_{\alpha}^{+}\left(\tau, \tau^{\prime}\right)=G^{+}\left(\chi(\tau), \chi\left(\tau^{\prime}\right)\right)$. In this notation we make explicit the fact that the Whightman function depends only on the difference of proper times and that the trajectory have a proper acceleration $\alpha$. In this way and by using the properties of the temporal ordered products, we obtain that the final state of the qubit in the interaction picture, eq. (A.23), is given by

$$
\rho_{I}(t)=\rho_{\mathrm{in}}+g^{2} \int_{-\bar{\tau}}^{t} d \tau \int_{-\bar{\tau}}^{t} d \tau^{\prime} \delta \rho\left(\tau, \tau^{\prime}\right) G_{\alpha}^{+}\left(\tau, \tau^{\prime}\right),
$$

where we have defined

$$
\delta \rho\left(\tau, \tau^{\prime}\right)=\delta \rho^{(a)}\left(\tau, \tau^{\prime}\right)+\delta \rho^{(b)}\left(\tau, \tau^{\prime}\right)+\delta \rho^{(b)}\left(\tau, \tau^{\prime}\right),
$$

with each term that corrects the state of the qubit given by

$$
\begin{aligned}
& \delta \rho^{(a)}\left(\tau, \tau^{\prime}\right)=m_{I}\left(\tau^{\prime}\right) \rho_{\mathrm{in}} m_{I}(\tau), \\
& \delta \rho^{(b)}\left(\tau, \tau^{\prime}\right)=-\theta(\Delta \tau) m_{I}(\tau) m_{I}\left(\tau^{\prime}\right) \rho_{\mathrm{in}}, \\
& \delta \rho^{(c)}\left(\tau, \tau^{\prime}\right)=-\theta(-\Delta \tau) \rho_{\mathrm{in}} m_{I}(\tau) m_{I}\left(\tau^{\prime}\right) .
\end{aligned}
$$

Then

$$
\begin{aligned}
& \delta \rho^{(a)}\left(\tau, \tau^{\prime}\right)=\left(\begin{array}{cc}
(1-p) e^{-i \omega \Delta \tau} & 0 \\
0 & p e^{i \omega \Delta \tau}
\end{array}\right) \\
& \delta \rho^{(b)}\left(\tau, \tau^{\prime}\right)=-\theta(\Delta \tau)\left(\begin{array}{cc}
p e^{i \omega \Delta \tau} & 0 \\
0 & (1-p) e^{-i \omega \Delta \tau}
\end{array}\right) \\
& \delta \rho^{(c)}\left(\tau, \tau^{\prime}\right)=-\theta(-\Delta \tau)\left(\begin{array}{cc}
p e^{i \omega \Delta \tau} & 0 \\
0 & (1-p) e^{-i \omega \Delta \tau}
\end{array}\right) .
\end{aligned}
$$

Therefore we can write

$$
\delta \rho\left(\tau, \tau^{\prime}\right)=\left((1-p) e^{-i \omega \Delta \tau}-p e^{i \omega \Delta \tau}\right) \sigma_{3},
$$


where $\sigma_{3}$ is the $Z$ Pauli matrix. Hence, we can write the final state of the qubit after interacted with the vacuum as

$$
\rho_{I}(t)=\rho_{\text {in }}+\delta p(t) \sigma_{3}
$$

where we have defined the change in the population of the excited state as

$$
\delta p(t)=g^{2} \int_{-\bar{\tau}}^{t} d \tau \int_{-\bar{\tau}}^{t} d \tau^{\prime}\left((1-p) e^{-i \omega \Delta \tau}-p e^{i \omega \Delta \tau}\right) G_{\alpha}^{+}\left(\tau, \tau^{\prime}\right)
$$

Therefore, after the interaction with the quantum vacuum, the density operator of the qubit is given by

$$
\rho_{I}(t)=\left(\begin{array}{cc}
p+\delta p(t) & 0 \\
0 & 1-(p+\delta p(t))
\end{array}\right) .
$$

One can see that the final increase of the population of the excited state $\delta p=\delta p(\bar{\tau})$, eq. (A.32), is caused by the interaction with the vacuum and this would give us a thermallike response, as predicted by Unruh [16]. We also realize that the change in the probability of the excited state depends also on the initial population of this state $p$, on the energy gap $\omega$ of the qubit system and also on the acceleration $\alpha$ of the qubit when interacting with the vacuum.

\section{B Regularization by Lorentzian profile}

In this appendix, we will show more explicitly how the vacuum fluctuations transfer heat to the qubit system. The integral that shows the connection between vacuum fluctuations and the specific trajectory of the qubit is given by

$$
\mathcal{J}(\alpha, \omega, \mathcal{T})=\int_{-\mathcal{T} / 2}^{\mathcal{T} / 2} d \tau \int_{-\mathcal{T} / 2}^{\mathcal{T} / 2} d \tau^{\prime} e^{i \omega \Delta \tau} G_{\alpha}^{+}\left(\tau, \tau^{\prime}\right)
$$

Here, the finite-time interval $\mathcal{T}$ is defined in terms of the velocity $v$ and the acceleration $\alpha$. This finite-time integral can be extended to infinite by using a regulator that is compact in the domain we are interested in and vanish outside. This procedure is in the same spirit of the regularization by a cut-off function of an infinite divergent integral. In particular, finite-time integrals related to the Unruh effect have been previously analyzed, e.g., in refs. $[24,25]$. However, since we are dealing with a kinematically closed trajectory there is no need of taking the limit of infinite time. Hence, we will identify eq. (B.1) with the expression

$$
\mathcal{J}(\alpha, \omega, \mathcal{T})=\int_{-\infty}^{\infty} d \tau \int_{-\infty}^{\infty} d \tau^{\prime} \xi_{\mathcal{T}}(\tau) \xi_{\mathcal{T}}\left(\tau^{\prime}\right) e^{i \omega \Delta \tau} G_{\alpha}^{+}\left(\tau, \tau^{\prime}\right)
$$

where $\xi_{\mathcal{T}}(\tau)$ is a compact smooth function that is nonvanishing for $-\mathcal{T} / 2<\tau<\mathcal{T} / 2$, being approximately zero outside that domain. We could try to adopt a Gaussian regulator of the kind $\xi_{\text {gauss }} \sim \exp \left(-\tau^{2} / \mathcal{T}^{2}\right)$. However, as we expand this Gaussian regulator to the complex plane $\tau \rightarrow z \in \mathbb{C}$, we see that it does not vanish for any $|z| \rightarrow \infty$. Actually, 


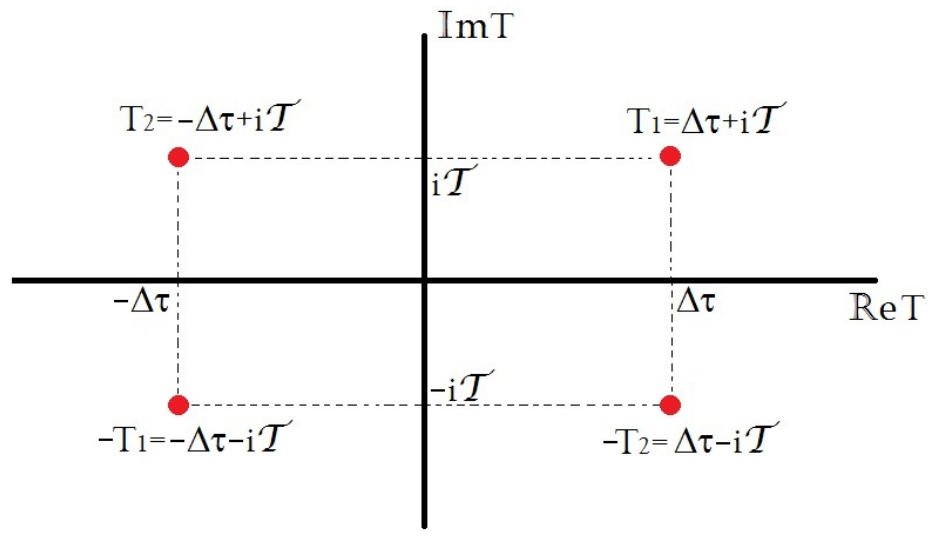

Figure 10. Pole structure of the regulator product $\xi_{\mathcal{T}}(\tau) \xi_{\mathcal{T}}\left(\tau^{\prime}\right)$ in the complex plane of $T=\tau+\tau^{\prime}$.

depending on the phase in $z=|z| e^{i \theta}$, this regulator can be divergent. In order to bypass this difficulty, we adopt as a regulator $\xi_{\mathcal{T}}(\tau)$ a Lorentzian profile rather than a Gaussian profile, i.e.

$$
\xi_{\mathcal{T}}(\tau)=\frac{(\mathcal{T} / 2)^{2}}{\tau^{2}+(\mathcal{T} / 2)^{2}}
$$

The expression above for $\xi_{\mathcal{T}}(\tau)$ yields

$$
\xi_{\mathcal{T}}(\tau) \xi_{\mathcal{T}}\left(\tau^{\prime}\right)=\frac{\mathcal{T}^{4}}{\left(T^{2}-T_{1}^{2}\right)\left(T^{2}-T_{2}^{2}\right)},
$$

where we have defined $T_{1}=\Delta \tau+i \mathcal{T}$ and $T_{2}=-\Delta \tau+i \mathcal{T}$. Hence, eq. (B.4) shows $\xi_{\mathcal{T}}(\tau) \xi_{\mathcal{T}}\left(\tau^{\prime}\right)$ explicitly as a function of $T=\tau+\tau^{\prime}$. This function posses a pole structure in the complex plane of $T$, as shown in figure 10. Naturally, the polar structure depends on $\Delta \tau=\tau-\tau^{\prime}$. Now, performing the change of integration variables from $\left(\tau, \tau^{\prime}\right)$ to $(T, \Delta \tau)$ one gets

$$
\mathcal{J}(\alpha, \omega, \mathcal{T})=\frac{1}{2} \int_{-\infty}^{\infty} d T \int_{-\infty}^{\infty} d(\Delta \tau) \xi_{\mathcal{T}}(\tau) \xi_{\mathcal{T}}\left(\tau^{\prime}\right) e^{i \omega \Delta \tau} G_{\alpha}^{+}(\Delta \tau)
$$

Here, we have made explicit the dependence on the Wightman correlation function of the scalar field evaluated at an accelerated trajectory, which is given by

$$
G_{\alpha}^{+}\left(\tau, \tau^{\prime}\right)=G_{\alpha}^{+}(\Delta \tau)=-\frac{\alpha^{2}}{16 \pi^{2}} \frac{1}{\sinh ^{2}\left(\frac{\alpha \Delta \tau}{2}-i \epsilon \alpha\right)}
$$

Therefore, one can see that the only dependence on $T$ is given by the regulator factors $\xi_{\mathcal{T}}(\tau) \xi_{\mathcal{T}}\left(\tau^{\prime}\right)$, such that

$$
\mathcal{J}(\alpha, \omega, \mathcal{T})=\frac{1}{2} \int_{-\infty}^{\infty} d(\Delta \tau) e^{i \omega \Delta \tau} G_{\alpha}^{+}(\Delta \tau) \int_{-\infty}^{\infty} d T \xi_{\mathcal{T}}(\tau) \xi_{\mathcal{T}}\left(\tau^{\prime}\right)
$$

Then, performing the integral on $T$ first, using the pole structure in figure 10 and the Cauchy-Riemann residue theorem of complex variables [36] one gets

$$
\int_{-\infty}^{\infty} d T \xi_{\mathcal{T}}(\tau) \xi_{\mathcal{T}}\left(\tau^{\prime}\right)=\frac{\pi}{2} \frac{\mathcal{T}^{3}}{\Delta \tau^{2}+\mathcal{T}^{2}}
$$



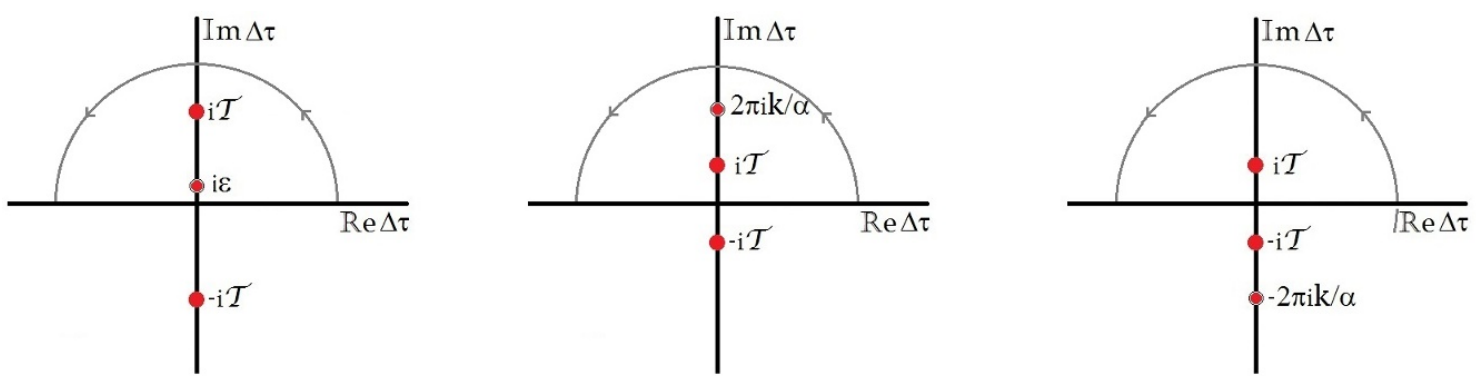

Figure 11. Pole structure in the complex plane of $\Delta \tau$ for the terms in eq. (B.11).

Putting this result into eq. (B.7) and using the alternative representation of the Wightman function on the accelerated trajectory [15]

$$
G_{\alpha}^{+}(\Delta \tau)=-\frac{1}{4 \pi^{2}} \sum_{k=-\infty}^{\infty} \frac{1}{(\Delta \tau-i \epsilon-2 \pi i k / \alpha)^{2}}
$$

we get that

$$
\mathcal{J}(\alpha, \omega, \mathcal{T})=-\frac{\mathcal{T}^{3}}{16 \pi} \int_{-\infty}^{\infty} d(\Delta \tau) \sum_{k=-\infty}^{\infty} \frac{e^{i \omega \Delta \tau}}{(\Delta \tau-i \epsilon-2 \pi i k / \alpha)^{2}\left(\Delta \tau^{2}+\mathcal{T}^{2}\right)}
$$

Splitting the term in the above sum, it follows that

$$
\begin{aligned}
\mathcal{J}(\alpha, \omega, \mathcal{T})= & -\frac{\mathcal{T}^{3}}{16 \pi} \int_{-\infty}^{\infty} d(\Delta \tau)\left(\frac{e^{i \omega \Delta \tau}}{(\Delta \tau-i \epsilon)^{2}\left(\Delta \tau^{2}+\mathcal{T}^{2}\right)}\right. \\
& +\sum_{k=0}^{\infty} \frac{e^{i \omega \Delta \tau}}{(\Delta \tau-2 \pi i k / \alpha)^{2}\left(\Delta \tau^{2}+\mathcal{T}^{2}\right)} \\
& \left.+\sum_{k=0}^{\infty} \frac{e^{i \omega \Delta \tau}}{(\Delta \tau+2 \pi i k / \alpha)^{2}\left(\Delta \tau^{2}+\mathcal{T}^{2}\right)}\right) .
\end{aligned}
$$

All the terms in the above expression have simple poles in $\pm i \mathcal{T}$. The first term corresponds to $k=0$ and possess a pole of second order at $i \epsilon$. In the second and third terms, we have neglected the $i \epsilon$ contribution, since in these terms the double pole is finite at $\pm 2 \pi i / \alpha$, respectively. This pole structure is shown in figure 11.

By using the Residue theorem and the definition of the transcendental Lerch-Hurwitz function

$$
\phi(z, s, a)=\sum_{k=0}^{\infty} \frac{z^{k}}{(k+a)^{s}}
$$

we obtain then that

$$
\begin{aligned}
\mathcal{J}(\alpha, \omega, \mathcal{T})= & \frac{(\alpha \mathcal{T} / 2)^{2} e^{-|\omega| \mathcal{T}}}{8 \sin ^{2}(\alpha \mathcal{T} / 2-i \epsilon)}+\frac{|\omega| \mathcal{T}}{4} \theta(\omega) \\
& +\frac{\alpha^{2} \mathcal{T}^{2} e^{-2 \pi|\omega| /|\alpha|}}{32 \pi^{2}}\left(\phi\left(e^{-2 \pi|\omega| /|\alpha|}, 2,1+\frac{|\alpha| \mathcal{T}}{2 \pi}\right)-\phi\left(e^{-2 \pi|\omega| /|\alpha|}, 2,1-\frac{|\alpha| \mathcal{T}}{2 \pi}\right)\right) \\
& +\frac{|\omega \alpha| \mathcal{T}^{2} e^{-2 \pi|\omega| /|\alpha|}}{16 \pi}\left(\phi\left(e^{-2 \pi|\omega| /|\alpha|}, 1,1+\frac{|\alpha| \mathcal{T}}{2 \pi}\right)-\phi\left(e^{-2 \pi|\omega| /|\alpha|}, 1,1-\frac{|\alpha| \mathcal{T}}{2 \pi}\right)\right),
\end{aligned}
$$


the small imaginary part $i \epsilon$ in the first term of the equation above can be iterpreted using the Cauchy principal value $1 /(x \mp i \epsilon)=(P / x) \pm i \pi \delta(x)$, see [15]. In this way, with $\epsilon>0$, we have that the function $\mathcal{J}$ tends to zero in the limit of vanishing interaction time $\mathcal{T}$. This function, eq. (B.13), apparently depends on three independent variables. However, as one can check, the combination between these variables leads us to a two-variable function. Indeed, if we define the function

$$
\begin{aligned}
J(x, y)= & \frac{(y / 2)^{2} e^{-|x| y}}{8 \sin ^{2}(y / 2)}-\frac{1}{8}+\frac{|x| y}{4} \theta(x) \\
& +\frac{y^{2} e^{-2 \pi|x|}}{32 \pi^{2}}\left(\phi\left(e^{-2 \pi|x|}, 2,1+\frac{y}{2 \pi}\right)-\phi\left(e^{-2 \pi|x|}, 2,1-\frac{y}{2 \pi}\right)\right) \\
& +\frac{|x| y^{2} e^{-2 \pi|x|}}{16 \pi}\left(\phi\left(e^{-2 \pi|x|}, 1,1+\frac{y}{2 \pi}\right)-\phi\left(e^{-2 \pi|x|}, 1,1-\frac{y}{2 \pi}\right)\right)
\end{aligned}
$$

then we can rewrite

$$
\mathcal{J}(\alpha, \omega, \mathcal{T})=J\left(\frac{\omega}{\alpha}, \alpha \mathcal{T}\right)
$$

By using this analytical expression, we can derive the general result shown in the appendix A. There, we obtained that the final general correction to the population of the excited state of the qubit due to the vacuum fluctuations is

$$
\delta p=g^{2} \int_{-\bar{\tau}}^{\bar{\tau}} d \tau \int_{-\bar{\tau}}^{\bar{\tau}} d \tau^{\prime}\left((1-p) e^{-i \omega \Delta \tau}-p e^{i \omega \Delta \tau}\right) G_{\alpha}^{+}\left(\tau, \tau^{\prime}\right)
$$

Then, using the definition of the integral in eq. (B.1) and also eq. (B.15), we can write

$$
\begin{aligned}
\delta p & =g^{2}((1-p) \mathcal{J}(\alpha,-\omega, 2 \bar{\tau})-p \mathcal{J}(\alpha, \omega, 2 \bar{\tau})), \\
& =g^{2}\left((1-p) J\left(-\frac{\omega}{\alpha}, 2 \alpha \bar{\tau}\right)-p J\left(\frac{\omega}{\alpha}, 2 \alpha \bar{\tau}\right)\right) .
\end{aligned}
$$

Defining the reduced acceleration of the qubit as the ratio $a=\alpha / \omega$ and taking into account that the interaction time of the qubit with the vacuum have to be such $\bar{\tau}=\operatorname{arctanh}(v) / \alpha$, we obtain

$$
\begin{aligned}
\delta p / g^{2} & =(1-p) J\left(-\frac{1}{a}, 2 \operatorname{arctanh} v\right)-p J\left(\frac{1}{a}, 2 \operatorname{arctanh} v\right) \\
& =(1-2 p) J\left(-\frac{1}{a}, 2 \operatorname{arctanh} v\right)-p \frac{\operatorname{arctanh} v}{2 a}
\end{aligned}
$$

In the final line we have used the property $J(x, y)-J(-x, y)=x y / 4$, which follows from eq. (B.14). This concludes the analysis of the regularized perturbative expression for the excitation correction $\delta p$.

Open Access. This article is distributed under the terms of the Creative Commons Attribution License (CC-BY 4.0), which permits any use, distribution and reproduction in any medium, provided the original author(s) and source are credited. 


\section{References}

[1] J. Gemmer, M. Michel and G. Mahler, Quantum Thermodynamics: Emergence of Thermodynamic Behavior Within Composite Quantum Systems, Lect. Notes Phys. 657, Springer-Verlag, Berlin Heidelberg, Germany (2004).

[2] J. Goold, M. Huber, A. Riera, L. del Rio and P. Skrzypczyk, The role of quantum information in thermodynamics - a topical review, J. Phys. A 49 (2016) 143001.

[3] K. Maruyama, F. Nori and V. Vedral, Colloquium: The physics of Maxwell's demon and information, Rev. Mod. Phys. 81 (2009) 1 [InSPIRE].

[4] H.E.D. Scovil and E.O. Schulz-DuBois, Three-level masers as heat engines, Phys. Rev. Lett. 2 (1959) 262.

[5] T.D. Kieu, The second law, Maxwell's demons, and work derivable from quantum heat engines, Phys. Rev. Lett. 93 (2004) 140403.

[6] T.D. Kieu, Quantum heat engines, the second law and Maxwell's daemon, Eur. Phys. J. D 39 (2006) 115.

[7] T. Zhang, W.-T. Liu, P.-X. Chen and C.-Z. Li, Four-level entangled quantum heat engines, Phys. Rev. A 75 (2007) 062102.

[8] G.-F. Zhang, Entangled quantum heat engines based on two two-spin systems with Dzyaloshinski-Moriya anisotropic antisymmetric inteaction, Eur. Phys. J. D 49 (2008) 123.

[9] H. Wang, S. Liu and J. He, Thermal entanglement in two-atom cavity QED and the entangled quantum Otto engine, Phys. Rev. E 79 (2009) 041113.

[10] J.M. Bardeen, B. Carter and S.W. Hawking, The four laws of black hole mechanics, Commun. Math. Phys. 31 (1973) 161 [inSPIRE].

[11] J.D. Bekenstein, Black holes and entropy, Phys. Rev. D 7 (1973) 2333 [InSPIRE].

[12] S.W. Hawking, Black hole explosions, Nature 248 (1974) 30 [INSPIRE].

[13] S.W. Hawking, Particle Creation by Black Holes, Commun. Math. Phys. 43 (1975) 199 [Erratum ibid. 46 (1976) 206] [INSPIRE].

[14] S.W. Hawking, Black Holes and Thermodynamics, Phys. Rev. D 13 (1976) 191 [InSPIRE].

[15] N.D. Birrell and P.C.W. Davis, Quantum Fields in Curved Space, Cambridge University Press, New York, U.S.A. (1982).

[16] W.G. Unruh, Notes on black hole evaporation, Phys. Rev. D 14 (1976) 870 [INSPIRE].

[17] S. Felicetti, C. Sabín, I. Fuentes, L. Lamata, G. Romero and E. Solano, Relativistic Motion with Superconducting Qubits, Phys. Rev. B 92 (2015) 064501 [arXiv:1503.06653] [INSPIRE].

[18] J. Rodríguez-Laguna, L. Tarruell, M. Lewenstein and A. Celi, Synthetic Unruh effect in cold atoms, Phys. Rev. A 95 (2017) 013627 [arXiv:1606.09505] [INSPIRE].

[19] J. Wang, Z. Tian, J. Jing and H. Fan, Quantum metrology and estimation of Unruh effect, Sci. Rep. 4 (2014) 7195.

[20] D.V. Ahluwalia, L. Labun and G. Torrieri, The Unruh effect and oscillating neutrinos, J. Phys. Conf. Ser. 706 (2016) 042006 [arXiv: 1505.04082] [InSPIRE]. 
[21] G. Cozzella, A.G.S. Landulfo, G.E.A. Matsas and D.A.T. Vanzella, Proposal for Observing the Unruh Effect using Classical Electrodynamics, Phys. Rev. Lett. 118 (2017) 161102 [arXiv: 1701.03446] [INSPIRE].

[22] L.C.B. Crispino, A. Higuchi and G.E.A. Matsas, The Unruh effect and its applications, Rev. Mod. Phys. 80 (2008) 787 [arXiv:0710.5373] [INSPIRE].

[23] B.S. DeWitt, General relativity, an Einstein centenary survey, S.W. Hawking and W. Israel eds., Cambridge University Press, U.K. (1979).

[24] B.F. Svaiter and N.F. Svaiter, Inertial and noninertial particle detectors and vacuum fluctuations, Phys. Rev. D 46 (1992) 5267 [INSPIRE].

[25] A. Higuchi, G.E.A. Matsas and C.B. Peres, Uniformly accelerated finite time detectors, Phys. Rev. D 48 (1993) 3731 [INSPIRE].

[26] M. Campisi, P. Hänggi and P. Talkner, Colloquium: Quantum fluctuations relations: Foundations and applications, Rev. Mod. Phys. 83 (2011) 771 [arXiv:1012.2268].

[27] R.A. Horn and C.R. Johnson, Matrix Analysis, 2nd Edition, Cambridge University Press, New York, NY, U.S.A. (2013).

[28] M. Born and V. Fock, Beweis des Adiabatensatzes, Z. Phys. 51 (1928) 165.

[29] T. Kato, On the adiabatic theorem of quantum mechanics, J. Phys. Soc. Jap. 5 (1950) 435.

[30] A. Messiah, Quantum mechanics, North-Holland, Amsterdam, The Netherlands (1962).

[31] W. Niedenzu, V. Mukherjee, A. Ghosh, A.G. Kofman, G. Kurizki, Quantum engine efficiency bound beyond the second law of thermodynamics, Nature Commun. 9 (2018) 165 [arXiv:1703.02911].

[32] J. Doukas, S.-Y. Lin, B.L. Hu and R.B. Mann, Unruh Effect under Non-equilibrium conditions: Oscillatory motion of an Unruh-DeWitt detector, JHEP 11 (2013) 119 [arXiv: 1307.4360] [INSPIRE].

[33] E.G. Brown and J. Louko, Smooth and sharp creation of a Dirichlet wall in $1+1$ quantum field theory: how singular is the sharp creation limit?, JHEP 08 (2015) 061 [arXiv: 1504.05269] [INSPIRE].

[34] G. Salton, R.B. Mann and N.C. Menicucci, Acceleration-assisted entanglement harvesting and rangefinding, New. J. Phys. 17 (2015) 035001.

[35] S. Deffner and E. Lutz, Nonequilibrium entropy productionfor open quantum systems, Phys. Rev. Lett. 107 (2011) 140404. Phys. Rev. Lett. 107 (2011) 140404 [arXiv:1103.4775].

[36] S.D. Fisher, Complex Variables, second edition, Dover, U.S.A. (1999). 